\title{
Forcing with Sequences of Models of Two Types
}

\author{
Itay Neeman
}

\begin{abstract}
We present an approach to forcing with finite sequences of models that uses models of two types. This approach builds on earlier work of Friedman and Mitchell on forcing to add clubs in cardinals larger than $\aleph_{1}$, with finite conditions. We use the two-type approach to give a new proof of the consistency of the proper forcing axiom. The new proof uses a finite support forcing, as opposed to the countable support iteration in the standard proof. The distinction is important since a proof using finite supports is more amenable to generalizations to cardinals greater than $\aleph_{1}$.
\end{abstract}

\section{Introduction}

There is by now a long history, tracing back to the work of Todorčević [11], of using finite increasing sequences of countable models as side conditions in forcing notions, to ensure properness of the resulting poset, and in particular to ensure that $\boldsymbol{\aleph}_{1}$ is not collapsed.

More recently Friedman [2] and Mitchell [6] independently discovered forcing notions that add clubs in $\theta>\aleph_{1}$ using finite conditions while preserving both $\aleph_{1}$ and $\theta$. The Friedman and Mitchell posets use countable models as side conditions to ensure preservation of the two cardinals. The side conditions are no longer increasing sequences; rather they are sets, with various agreement and coherence conditions on the models in them.

In this paper we reformulate this approach using models of two types, countable and transitive, rather than just countable. This allows us to return to a situation where side conditions are increasing sequences, simplifying the definition of the poset of side conditions.

We show how the resulting poset can be used for the initial Friedman and Mitchell applications, for an additional application which involves collapsing cardinals in con-

Received October 9, 2011; accepted September 20, 2012

2010 Mathematics Subject Classification: Primary 03E35

Keywords: forcing, finite conditions, proper forcing axiom

(C) 2014 by University of Notre Dame 10.1215/00294527-2420666 
texts where it is important not to add branches to certain trees in $V$, and most importantly for a new proof of the consistency of the proper forcing axiom.

The original proof of the consistency of PFA used preservation of properness under countable support iterations. The use of countable support makes it impossible to apply similar ideas for forcing axioms that involve meeting more than $\aleph_{1}$ dense sets (in posets that admit master conditions for more than countable structures). The proof we give uses finite support, and instead of appealing to preservation of properness, which fails for finite support iterations, it incorporates the two-type side conditions into the iteration, using them to ensure preservation of $\aleph_{1}$ and of a supercompact cardinal that becomes $\boldsymbol{\aleph}_{2}$. This finite support proof has analogues that yield forcing axioms for meeting more than $\aleph_{1}$ dense sets, but these will be handled in a separate paper.

The poset of sequences of models of two types is presented in Section 2. We present it in greater generality than we need. For most applications we only need the following finite version. Let $K$ be a structure satisfying a large enough fragment of ZFC. Let $\delta$ be a collection of countable $M \prec K$ with $M \in K$. Let $\mathcal{T}$ be a collection of transitive $W \prec K$ with $W \in K$. Suppose that $M \cap W \in W$ and $M \cap W \in \delta$ whenever $M \in \delta, W \in \mathcal{T}$, and $W \in M$. (This can be arranged in many different settings, for example, if all elements of $\mathcal{T}$ are countably closed and $\delta$ consists of all countable elementary substructures of $K$.) Conditions in the finite two-type model sequence poset associated to $\delta$ and $\mathcal{T}$ are simply $\in$-increasing sequences of models from $\delta \cup \mathcal{T}$, closed under intersections. More precisely, a condition is a sequence $s$

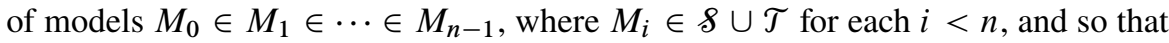
for every $i, j<n$, the intersection $M_{i} \cap M_{j}$ appears in the sequence. Conditions are ordered by reverse inclusion.

For $Q$ a model that appears in $s$, the residue of $s$ in $Q$, denoted $\operatorname{res}_{Q}(s)$, is the subsequence of $s$ consisting of models of $s$ that belong to $Q$. We prove that the residue is itself a condition (meaning that it is $\in$-increasing and closed under intersections). We also prove that if $t \in Q$ is a condition that extends $\operatorname{res}_{Q}(s)$, then $s$ and $t$ are compatible. This is the most important result proved in Section 2. It allows deducing that the poset of two-type model sequences is strongly proper, in a sense defined by Mitchell [6].

The basics of strong properness are presented in Section 3 and are connected to the poset of two-type model sequences in Section 4. Then in Section 5 we present the initial applications, yielding the models of Friedman and Mitchell and the method for collapsing cardinals to $\kappa^{+}$without adding branches of length $\kappa^{+}$through trees in $V$.

Finally, in Section 6 we use the two-type model sequences to prove the consistency of PFA with finite supports.

The main definitions and results in this paper, including the finite two-type model sequences and their use for a finite support proof of the consistency of PFA, were presented in Neeman [9], which also goes further and indicates how forcing with finite conditions helps in obtaining higher analogues of the proper forcing axiom. Since then there have already been some applications of the two-type model sequences, for example, by Veličković and Venturi [13], using side conditions to obtain new proofs of results of Koszmider, adding a chain of length $\omega_{2}$ in $\left(\omega_{1}^{\omega_{1}},<_{\text {Fin }}\right)$, and of Baumgartner and Shelah, adding a thin, very tall superatomic Boolean algebra. Earlier applications of the Friedman and Mitchell side conditions include Friedman [3], 
showing that PFA does not imply that a model correct about $\boldsymbol{\aleph}_{2}$ must contain all reals, and Mitchell [7], showing that $I\left(\omega_{2}\right)$ can be trivial.

\section{The Model Sequence Poset}

Fix cardinals $\kappa<\lambda$. Typically in uses later on $\lambda$ will be the successor of $\kappa$. Most often in fact we will take $\kappa=\omega$ and $\lambda=\omega_{1}$. Fix a transitive set $K$ so that $\kappa, \lambda \in K$ and $(K ; \in)$ satisfies some large enough fragment of ZFC. More precisely, we need enough of ZFC in $(K ; \in)$ to imply that $K$ is closed under the pairing, union, intersection, set difference, Cartesian product, and transitive closure operations, closed under the range and restriction operations on functions, and that for each $x \in K$, the closure of $x$ under intersections belongs to $K$, there is a bijection from an ordinal onto $x$ in $K$, and there is a sequence in $K$ consisting of the members of $x$ arranged in nondecreasing von Neumann rank. Typically in applications we will take $K=H(\theta)$ for a regular cardinal $\theta$; these closure properties then hold for $K$.

Remark 2.1 Our main applications of the results in this section will all be with $\kappa=\omega$ and $\lambda=\omega_{1}$. Many of the definitions and claims in this section are simpler in this case. We will point out some of the simplifications, mainly to the definitions, in remarks throughout the section. Most of the simplifications in the case $\kappa=\omega$ are due to the fact that all models are closed under finite sequences. Similar simplifications hold for $\kappa>\omega$ if we restrict to models that are $<\kappa$-closed.

Definition 2.2 $\quad \&$ and $\mathcal{T}$ are appropriate for $\kappa, \lambda$, and $K$ if we have the following.

(1) $\mathcal{T}$ is a collection of transitive $W \prec K$, and $\delta$ is a collection of $M \prec K$ with $\kappa \subseteq M$ and $|M|<\lambda$. All elements of $\delta \cup \mathcal{T}$ belong to $K$ and contain $\{\kappa, \lambda\}$.

(2) If $M_{1}, M_{2} \in \delta$ and $M_{1} \in M_{2}$, then $M_{1} \subseteq M_{2}$.

(3) If $W \in \mathcal{T}, M \in \delta$, and $W \in M$, then $M \cap W \in W$ and $M \cap W \in \delta$.

(4) Each $W \in \mathcal{T}$ is closed under sequences of length $<\kappa$ in $K$.

Remark 2.3 In the case where $\kappa=\omega$ and $\lambda=\omega_{1}$, condition (1) simplifies to requiring that elements of $\mathcal{T}$ and $\delta$ be, respectively, transitive and countable elementary submodels of $K$ that belong to $K$. Moreover, conditions (2) and (4) can be dropped in this case, as they follow from the elementarity in condition (1).

Suppose that $\delta$ and $\mathcal{T}$ are appropriate. We define a poset $\mathbb{P}=\mathbb{P}_{\kappa, \delta, \mathcal{T}, K}$ that we call the poset of two-type model sequences, or simply the sequence poset, associated to $\kappa, \delta, \mathcal{T}$, and $K$. Conditions are sequences of models in $\delta \cup \mathcal{T}$, satisfying certain requirements that we specify in the next definition.

Definition 2.4 A condition in $\mathbb{P}$ is a sequence $\left\langle M_{\xi} \mid \xi<\gamma\right\rangle$ of length $\gamma<\kappa$, which belongs to $K$, and is such that the following hold.

(1) For each $\xi, M_{\xi}$ is either an element of $\mathcal{T}$ or an element of $\delta$.

(2) The sequence is increasing in the following sense: for each $\zeta<\gamma$, the set $\left\{\xi<\zeta \mid M_{\xi} \in M_{\zeta}\right\}$ is cofinal in $\zeta$. In particular, for successor ordinals $\zeta<\gamma, M_{\zeta-1} \in M_{\zeta}$.

(3) For each $\zeta<\gamma$, the sequence $\left\langle M_{\xi} \mid \xi<\zeta \wedge M_{\xi} \in M_{\zeta}\right\rangle$ belongs to $M_{\zeta}$.

(4) The sequence is closed under intersections, meaning that for all $\zeta$, $\xi<\gamma$, $M_{\zeta} \cap M_{\xi}$ is on the sequence. 
Note that condition (3) holds automatically for transitive nodes $M_{\zeta}$. It follows for such nodes from the closure of $M_{\zeta}$ in $K$, the fact that the entire sequence $\left\langle M_{\xi} \mid \xi<\gamma\right\rangle$ belongs to $K$, and enough closure for $K$ to produce $\left\langle M_{\xi} \mid \xi<\zeta \wedge M_{\xi} \in M_{\zeta}\right\rangle$ from $M_{\zeta}$ and $\left\langle M_{\xi} \mid \xi<\gamma\right\rangle$.

Remark 2.5 In the case where $\kappa=\omega$ and $\lambda=\omega_{1}$, condition (3) of Definition 2.4 and the requirement of membership in $K$ hold automatically, and condition (2) requires simply that $M_{\zeta-1} \in M_{\zeta}$ for each $\zeta>0$. Thus the definition in this case simplifies to the following: $\left\langle M_{\xi} \mid \xi<\gamma\right\rangle$ is a finite $\in$-increasing sequence of elements of $\delta \cup \mathcal{T}$ and is closed under intersections.

Claim 2.6 If $\xi<\zeta$, then $M_{\xi}$ has smaller von Neumann rank than $M_{\zeta}$.

Proof This is immediate by induction on $\zeta$ using condition (2).

Abusing notation slightly, we often refer to a condition as a set $\left\{M_{\xi} \mid \xi<\gamma\right\}$ rather than a sequence. There is no loss of information in talking about the set rather than the sequence, since by Claim 2.6 the sequence order is determined uniquely from the elements of the sequence.

Definition 2.7 Conditions in $\mathbb{P}$ are ordered by reverse inclusion (with conditions viewed as sets). In other words, $\left\{M_{\xi} \mid \xi<\gamma\right\} \leq\left\{N_{\xi} \mid \xi<\delta\right\}$ iff $\left\{M_{\xi} \mid \xi<\gamma\right\} \supseteq\left\{N_{\xi} \mid \xi<\delta\right\}$.

Claim 2.8 Suppose that $\kappa$ is regular, let $\tau \leq \kappa$ be regular, and suppose that $K$ and all models in 8 are $<\tau$-closed in $V$. Then $\mathbb{P}$ is $<\tau$-closed.

Proof If $\kappa$ is regular and $K$ and all models in $\delta$ are $<\tau$-closed, then the union of a decreasing set of fewer than $\tau$ conditions in $\mathbb{P}$ is itself, when ordered by von Neumann rank, a condition in $\mathbb{P}$. This is immediate from the definitions. The closure is needed for condition (3) in Definition 2.4.

We refer to elements of $\mathcal{T} \cup \mathcal{S}$ as nodes. By condition (1) in Definition 2.2, $\mathcal{S}$ and $\mathcal{T}$ are disjoint, so each node belongs to exactly one of them. Elements of $\mathcal{T}$ are transitive nodes, also called nodes of transitive type. Elements of 8 are small nodes, or nodes of small type. We say that $M$ is of the same or smaller type than $N$ if the two nodes are of the same type or $M$ is of small type and $N$ is of transitive type. Given a condition $s=\left\{M_{\xi} \mid \xi<\gamma\right\}$ and nodes $M=M_{\xi}$ and $N=M_{\zeta}$ which belong to $s$, we use interval notation in the natural way; for example, $(M, N)$ is the interval of nodes strictly between $M$ and $N$, namely, the interval of nodes $M_{\iota}, \xi<\iota<\zeta$.

Definition 2.9 $s$ is a precondition if it satisfies conditions (1) and (2) in Definition $2.4 ; s$ is a nice precondition if it also satisfies condition (3).

In the case where $\kappa=\omega$ and $\lambda=\omega_{1}$, a precondition is a finite $\epsilon$-increasing sequence from $\delta \cup \mathcal{T}$, and niceness holds automatically by closure of all models under finite sequences.

Claim 2.10 Let $s$ be a precondition.

(1) If $M$ and $Q$ are nodes in $s$, with $M \in Q$ and $M$ of the same or smaller type than $Q$, then $M \subseteq Q$.

(2) If $W$ is a node in $s$ of transitive type and $M$ is a node in s occurring below $W$, then $M \in W$, and hence by (l) also $M \subseteq W$. 
(3) If $Q \in s$ is of small type, $M \in s$ occurs before $Q$, and there are no nodes of transitive type in $s$ between $M$ and $Q$, then $M \in Q$. If in addition $M$ is of small type, then by ( 1 ) also $M \subseteq Q$.

Proof The first condition is clear, by conditions (1) and (2) in Definition 2.2.

For condition (2), let $W \in s$ be of transitive type, and let $M$ occur below $W$. By condition (2) of Definition 2.4, there are nodes of $s$ at or above $M$ which belong to $W$. Let $M^{*}$ be the least one. By condition (1) of the claim, $M^{*} \subseteq W$. By minimality of $M^{*}$ and condition (2) of Definition 2.4 it follows that $M^{*}$ must be equal to $M$, and hence $M=M^{*} \in W$.

Fix finally $Q$ and $M$ as in condition (3). Again there are nodes of $s$ at or above $M$ which belong to $Q$. Let $M^{*}$ be the first one. If $M^{*}$ is $M$, then $M \in Q$, and we are done, so suppose that $M^{*}$ occurs above $M$. In this case by assumption of the condition, $M^{*}$ is of small type, and hence by condition (1), $M^{*} \subseteq Q$. As in the previous paragraph, the minimality of $M^{*}$ now implies that $M^{*}$ is $M$.

Claim 2.11 Let $s$ be a precondition. Let $M_{1}$ and $M_{2}$ be nodes of $s$ with $M_{1}$ occurring before $M_{2}$. Suppose that there are nodes of transitive type between $M_{1}$ and $M_{2}$. Then there are nodes of transitive type between them that belong to $M_{2}$.

Proof Work by induction on $M_{2}$. Let $W$ be a node of transitive type between $M_{1}$ and $M_{2}$. Using condition (2) of Definition 2.4, there is $W^{*}$, occurring at or above $W$, that belongs to $M_{2}$. If $W^{*}$ is of transitive type, we are done. Otherwise by Claim 2.10, $W^{*} \subseteq M_{2}$. By induction there is a node of transitive type between $M_{1}$ and $W^{*}$ that belongs to $W^{*}$. Since $W^{*} \subseteq M_{2}$, the node belongs to $M_{2}$.

Claim 2.12 Let $s$ be a precondition. Suppose that $s$ satisfies the following weak closure under intersections:

(w4) If $W$ and $M$ are nodes in s of transitive and small type, respectively, and $W \in M$ (in particular, $W$ occurs before $M$ ), then $M \cap W$ is a node in s.

Then $s$ is closed under intersections; in other words, it satisfies condition (4) in Definition 2.4.

Proof Suppose that $s$ is not closed under intersections, and let $Q$ and $M$ witness this, with $Q$ occurring before $M$, and $M$ minimal.

If $M$ is of transitive type, then by Claim 2.10, $Q \subseteq M$; hence $M \cap Q=Q$ is a node of $s$. So we may assume that $M$ is of small type.

If there are no nodes of transitive type between $Q$ and $M$, then by Claim 2.10, $Q \in M$. If $Q$ is of small type, then by the same claim $Q \subseteq M$, so $M \cap Q=Q$ is a node of $s$. If $Q$ of transitive type, then by condition (w4) $M \cap Q$ is a node of $s$.

So we may assume that there are nodes of transitive type between $Q$ and $M$. By Claim 2.11 there is such a node $W$ with $W \in M$. By condition (w4) then $M \cap W$ is a node of $s$. It must occur before $W$ and therefore before $M$. By induction then $(M \cap W) \cap Q$ is a node of $s$. Since $W$ is a node of transitive type above $Q$, we have $Q \subseteq W$ by Claim 2.10. Hence $M \cap Q=(M \cap W) \cap Q$, so $M \cap Q$ is a node of $s$.

Definition 2.13 Let $s$ be a precondition, and let $Q$ be a node of $s$. The residue of $s$ in $Q$, denoted $\operatorname{res}_{Q}(s)$, is the set $\{M \in s \mid M$ belongs to $Q\}$.

Claim 2.14 If $s$ is a nice precondition, then $\operatorname{res}_{Q}(s)$ belongs to $Q$. 
Proof This is immediate from the definition of a nice precondition.

Claim 2.15 Let $s$ be a condition. Let $Q \in s$ be of small type, and let $W \in \operatorname{res} Q(s)$ be of transitive type. Then there are no nodes of $\operatorname{res}_{Q}(s)$ in the interval $[Q \cap W, W)$ of $s .(Q \cap W$ belongs to $s$ by closure of $s$ under intersections.)

Proof Let $N$ be a node of $\operatorname{res}_{Q}(s)$ that occurs before $W$ in $s$. Then since $W$ is of transitive type, $N \in W$ by Claim 2.10. Since $N \in \operatorname{res} Q(s), N \in Q$ by definition of the residue. So $N \in Q \cap W$, and this implies that $N$ can only occur before $Q \cap W$ in $s$.

Definition 2.16 Let $s$ be a condition, and let $Q \in s$ be of small type. Let $W$ be a node of transitive type in $\operatorname{res}_{Q}(s)$. Then the interval $[Q \cap W, W)$ of $s$ is called a residue gap of $s$ in $Q$.

Claim 2.17 Let $s$ be a condition, and let $Q$ be a node of $s$. If $Q$ is of transitive type, then $\operatorname{res}_{Q}(s)$ consists of all nodes of $s$ that occur before $Q$. If $Q$ is of small type, then $\operatorname{res} Q(s)$ consists of all nodes of $s$ that occur before $Q$ and do not belong to residue gaps of $s$ in $Q$.

Proof If $Q$ is of transitive type, then by Claim 2.10 all nodes of $s$ before $Q$ belong to $Q$ and are therefore nodes of the residue. Nodes from $Q$ upward have equal or higher von Neumann ranks than $Q$ and therefore cannot belong to $Q$, so they are not nodes of the residue.

Suppose that $Q$ is of small type. Again nodes occurring from $Q$ upward cannot belong to $Q$ because they have the same or higher von Neumann rank, so they are not nodes of the residue. Nodes below $Q$ that belong to residue gaps are not in the residue by Claim 2.15. It remains to prove that all nodes below $Q$ that are outside residue gaps belong to the residue.

Let $N$ be a node of $s$ below $Q$, outside all residue gaps. If there are no transitive nodes between $N$ and $Q$, then by Claim 2.10, $N$ belongs to $Q$ and therefore $N$ belongs to the residue. Suppose that there are transitive nodes between $N$ and $Q$. By Claim 2.11 there are such nodes that belong to $Q$. Let $W$ be the first one. Then $[Q \cap W, W]$ is a residue gap. Since $N$ occurs below $W$ and outside all residue gaps, it must occur below $Q \cap W$. By minimality of $W$ there are no transitive nodes between $N$ and $Q \cap W$ that belong to $Q \cap W$, and hence by Claim 2.11 there are outright no transitive nodes between $N$ and $Q \cap W$. By Claim 2.10 then $N \in Q \cap W$; hence in particular $N \in Q$, and therefore $N$ belongs to the residue.

Lemma 2.18 Let $s$ be a condition, and let $Q$ be a node of $s$. Then $\operatorname{res}_{Q}(s)$ is a condition.

Proof If $Q$ is of transitive type, then $\operatorname{res}_{Q}(s)$ is an initial segment of $s$ and is easily seen to be a condition. Suppose then that $Q$ is of small type. We prove that the residue satisfies the conditions in Definition 2.4.

The residue belongs to $K$ since it can be obtained from $Q$ and $s$ using simple set operations. Condition (1) of Definition 2.4 for the residue is immediate from the same condition for $s$. Condition (4) for the residue is again immediate, from the same condition for $s$ and the elementarity of $Q$ in $K$ (which implies that the intersection of two nodes that belong to $Q$ is itself an element of $Q$ ). Conditions (2) and (3) are clear if $M_{\zeta} \in \operatorname{res}_{Q}(s)$ is of small type, since in this case $M_{\zeta} \subseteq Q$ and any node 
of $s$ that belongs to $M_{\zeta}$ is also a node of the residue. Finally, if $M_{\zeta} \in \operatorname{res}_{Q}(s)$ is of transitive type, then all nodes of $s$ (and of $\operatorname{res}_{Q}(s)$ ) that occur before $M_{\zeta}$ are elements of $M_{\zeta}$, by Claim 2.10. Condition (2) for the residue at $\zeta$ follows immediately from this. Condition (3) holds automatically since $M_{\zeta}$ is of transitive type.

Definition 2.19 Two conditions $s$ and $t$ are compatible in $\mathbb{P}$ if there is a condition $r$ such that $r \supseteq s \cup t$. The conditions are directly compatible if $r$ can be taken to be exactly the closure of $s \cup t$ under intersections.

Lemma 2.20 Let $s$ be a condition, and let $Q \in s$ be a transitive node. Suppose that $t$ is a condition that belongs to $Q$ and extends $\operatorname{res}_{Q}(s)$. Then $s \cup t$ is a condition, and in particular $s$ and $t$ are directly compatible.

Proof $\quad K$ satisfies enough of ZFC that $s \cup t \in K$. Condition (1) of Definition 2.4 is immediate for $s \cup t$. Condition (2) too is clear, using the fact that $s \cup t$ is the same as $s$ above $Q$ and the same as $t$ below $Q$. For condition (4), it is enough by Claim 2.12 to verify (w4) of the claim. Fix nodes $W \in M$ of transitive and small type, respectively, in $s \cup t$. If $M$ occurs below $Q$, then both $M$ and $W$ are nodes of $t$, and closure of $t$ under intersections implies that $M \cap W$ is a node of $t$ and hence of $s \cup t$. If $M$ and $W$ both occur at or above $Q$, then both are nodes of $s$, and closure of $s$ implies that $M \cap W$ is a node of $s$ and hence of $s \cup t$. If $M$ occurs above $Q$ and $W$ occurs below $Q$, then by Claim 2.10, $W \subseteq Q$, so $M \cap W=M \cap Q \cap W$. $M \cap Q$ is a node of $s$ below $Q$ and hence a node of res $Q(s)$, hence a node of $t$. W is also a node of $t$. By closure of $t$ under intersections it follows that $M \cap Q \cap W$ is a node of $t$ and hence a node of $s \cup t$.

Finally, condition (3) for $s \cup t$ is clear if $M_{\zeta}$ occurs below $Q$, since the part of $s \cup t$ below $Q$ is simply $t$. The condition is also clear if $M_{\zeta}=Q$, since $Q$ is transitive. If $M_{\zeta}$ occurs above $Q$, then $\left\{N \in s \cup t \mid N \in M_{\zeta}\right\}=\left\{N \in s \mid N \in M_{\zeta}\right\} \cup\{N \in t \mid$ $\left.N \in M_{\zeta} \cap Q\right\}$. The left part of the union belongs to $M_{\zeta}$ since $s$ is a condition, and the right part of the union belongs to $M_{\zeta}$ (in fact to $M_{\zeta} \cap Q$ ) since $t$ is a condition and $M_{\zeta} \cap Q$ is a node of $t$. The entire union then belongs to $M_{\zeta}$ by elementarity of $M_{\zeta}$ in $K$.

Lemma 2.21 Let $s$ be a condition, and let $Q \in s$ be a small node. Suppose that $t$ is a condition that belongs to $Q$ and extends $\operatorname{res}_{Q}(s)$. Then $s$ and $t$ are directly compatible.

Proof We first show that $s \cup t$ is increasing. (The proof of this will use the assumption that $s$ is closed under intersections.) We then add nodes to $s \cup t$ that close it under intersections, and we show that the sequence resulting from the addition of these nodes is a condition. Since the sequence we generate is obtained from $s$ and $t$ using simple set operations (union, and closure under intersections), it belongs to $K$. So we will only have to worry about the other clauses in Definition 2.4.

Claim 2.22 $s \cup t$ is increasing, and is therefore a precondition.

Proof Let $u$ consist of the nodes of $s$ above $Q$. It is clear that $t \cup\{Q\} \cup u$ is increasing or, more precisely, that it satisfies condition (2) of Definition 2.4 when ordered in the natural way, namely, the nodes of $t$ ordered as they are in $t$, followed by $Q$, followed by the nodes of $u$ ordered as they are in $s$. The reason is that $t$ is a condition and hence increasing; $t \in Q$, and hence $t \subseteq Q$ by condition (1) in 
Definition 2.2 and the fact that $|t|<\kappa$, and $\{Q\} \cup u$ is a tail end of the condition $s$ and hence increasing.

Since $t$ extends res $Q(s)$, by Claim 2.17, the only nodes of $s$ that do not belong to $t \cup\{Q\} \cup u$ are the nodes in residue gaps of $s$ in $Q$. Recall that residue gaps are intervals in $s$ of the form [ $Q \cap W, W$ ), where $W$ is a transitive node of $s$ that belongs to $Q$. In particular, $W$ belongs to $t$ and hence to $t \cup\{Q\} \cup u$.

We prove that the sequence obtained from $t \cup\{Q\} \cup u$ by adding the nodes of each residue gap $[Q \cap W, W)$, immediately before $W$ and ordered inside the interval according to their ordering in $s$, is increasing. Since the resulting sequence has all nodes of $s \cup t$, this establishes the claim.

Since $t \cup\{Q\} \cup u$ is increasing, and each residue gap is increasing (being a segment of a condition), it is enough to check condition (2) of Definition 2.4 at the borders of each residue gap $[Q \cap W, W$ ). At the higher border the condition follows from the same condition for $s$ at $W$, since the residue gap includes a tail end of nodes of $s$ below $W$. At the lower end the condition follows from the fact that $t$ is increasing and contained in $Q$. Since $t$ is increasing, the set of nodes of $t$ which belong to $W$ is cofinal below $W$. Since $t$ is contained in $Q$, all these nodes belong to $Q \cap W$.

Remark 2.23 It follows from the proof that for any residue gap [ $Q \cap W, W$ ) of $s$ in $Q$, no nodes of $t$ occur in the interval $[Q \cap W, W$ ) of $s \cup t$.

Let $W$ be a transitive node of $t$ which does not belong to $s$. Suppose that there are transitive nodes of $s$ in the interval $(W, Q)$ of $s \cup t$, and let $W^{*}$ be the first one. Let $E_{W}$ list, in order, the small nodes of $s$ starting from $Q \cap W^{*}$, up to but not including the first transitive node of $s$ above $Q \cap W^{*}$.

Note that $W \in M$ for each $M \in E_{W}$. This is certainly the case for the first element of $E_{W}$, namely, $Q \cap W^{*}$, since $W \in W^{*}$ by Claim 2.10, and $W \in t \subseteq Q$. For $M$ occurring above $Q \cap W^{*}$ in $E_{W}, W \in Q \cap W^{*} \subseteq M$, where the final inclusion holds by Claim 2.10 as all nodes of $s$ between $Q \cap W^{*}$ and $M$ are small.

Define $F_{W}$ to be $\left\{M \cap W \mid M \in E_{W}\right\}$, with the ordering induced by the ordering of nodes in $E_{W}$. Since $M$ is small and $W \in M$ for each $M \in E_{W}$, each element $M \cap W$ of $F_{W}$ is a small node by Definition 2.2. It is easy to check that $F_{W}$ is increasing, and indeed $M \cap W \in M^{\prime} \cap W$ whenever $M$ occurs below $M^{\prime}$ in $E_{W}$. The lowest node of $F_{W}$ is $\left(Q \cap W^{*}\right) \cap W=Q \cap W$, and by Definition 2.2, all nodes of $F_{W}$ belong to $W$.

Let $W$ be a transitive node of $t$ which does not belong to $s$, and suppose that there are no transitive nodes of $s$ in the interval $(W, Q)$. Let $E_{W}$ list, in order, the small nodes of $s$ starting from $Q$, up to but not including the first transitive node of $s$ above $Q$ if there is one, and all nodes of $s$ starting from $Q$ if there are no transitive nodes above $Q$.

Again, $W \in M$ for each $M \in E_{W}$, since $W \in Q \subseteq M$, with the final inclusion using Claim 2.10. Again define $F_{W}$ to be $\left\{M \cap W \mid M \in E_{W}\right\}$, with the ordering induced by the ordering of the nodes in $E_{W}$. Again by Definition 2.2, all elements of $F_{W}$ are small nodes that belong to $W$. The first node of $F_{W}$ is $Q \cap W$, and again $F_{W}$ is increasing.

Let $r$ be obtained from $s \cup t$ by adding all nodes in $F_{W}$, for each transitive $W$ that belongs to $t-s$, placing the nodes of $F_{W}$ in order, right before $W$. We will prove that with this specific placement, $r$ is increasing. 
Remark 2.24 Note that every node of $r$ that belongs to $Q$ is a node of $t$. This is certainly the case for nodes of $s$, since $t$ extends $\operatorname{res}_{Q}(s)$. The only other nodes of $r$ which need to be checked are the nodes in $F_{W}$ for transitive $W \in t-s$, but these do not belong to $Q$ : They have the form $M \cap W$ with $M \in E_{W}$, which implies that $M$ is a small node that contains either $Q$ or $Q \cap W^{*}$, for $W^{*}$ transitive above $W$. Either way $M \cap W$ contains $Q \cap W$, which is impossible when $M \cap W \in Q$ (since then $M \cap W \in Q \cap W)$.

Claim 2.25 The sequence $r$ is increasing, and is therefore a precondition.

Proof Since $s \cup t$ is increasing, and each added interval $F_{W}$ is increasing, it is enough to verify condition (2) of Definition 2.4 at the borders of each added interval $F_{W}$.

At the upper border, every element of $F_{W}$ belongs to $W$. For the lower border, we have to show that cofinally many elements of $r$ below $Q \cap W$ belong to $Q \cap W$. Since all elements of $t$ below $W$ belong to $Q \cap W$ (as $t \subseteq Q$ ), it is enough to check that $t$ is cofinal in $r$ below $Q \cap W$. To check this, note that the only elements of $r$ below $Q \cap W$ which do not belong to $t$ are either in (a) residue gaps $[Q \cap \bar{W}, \bar{W}$ ) of $s$ in $Q$, or (b) added intervals $F_{\bar{W}}$. The gap or added interval cannot overlap $W$, since $W$ itself is a node of $t$. (Residue gaps do not include nodes of $t$ by Remark 2.23.) So in both (a) and (b), if the gap or added interval has nodes below $Q \cap W$, it must be that $\bar{W}$ occurs before $W$. Since $\bar{W}$ is a node of $t$, in both cases the gap or added interval is capped by a node of $t$ below $Q \cap W$. It follows that the nodes of $t$ are cofinal in $r$ below $Q \cap W$.

\section{Claim 2.26 The set of nodes of $r$ is closed under intersections.}

Proof It is enough to verify condition (w4) of Claim 2.12. Fix then $W \in M$ of transitive and small type, respectively, both nodes of $r$. We prove that $M \cap W$ is a node of $r$.

Since the intervals added to $s \cup t$ to form $r$ consist only of small nodes, $W$ must be a node of $s \cup t$. Suppose first that it is a node of $s$.

If $W$ occurs above $Q$, then $M$ must also occur above $Q$; hence $M$ is a node of $s$, and $M \cap W$ is a node of $s$ by closure of $s$ under intersections. Suppose then that $W$ occurs below $Q$. If $M$ is a node of $s$, then $M \cap W$ is a node of $s$ and hence of $r$. If $M$ is a node of one of the intervals added to $s \cup t$ to form $r$, then it is of the form $M^{\prime} \cap W^{*}$ for some $M^{\prime} \in s$ and $W^{*} \in t$. $W^{*}$ is above $M$, hence above $W$, so $W \subseteq W^{*}$. Then $M \cap W=\left(M^{\prime} \cap W^{*}\right) \cap W=M^{\prime} \cap W \in s$ where membership of $M^{\prime} \cap W$ in $s$ follows from the closure of $s$, as both $M^{\prime}$ and $W$ are nodes of $s$. The last remaining possibility (in the case when $W$ is a node of $s$ ) is that $M$ is a node of $t$. Then $M \in Q$, and since $M$ is small it follows that $M \subseteq Q$, so $W \in Q$. $W$ is then a node of $\operatorname{res}_{Q}(s)$ and hence a node of $t$. By closure of $t, M \cap W$ is a node of $t$ and hence of $r$.

Consider next the case when $W$ is not a node of $s$. In other words, it is a node of $t-s$. If $M$ is a node of $t$, then $M \cap W$ is a node of $t$ and hence of $r$. If $M$ is a node of one of the intervals added to $s \cup t$ to form $r$, then it is of the form $M^{\prime} \cap W^{\prime}$ for some $M^{\prime} \in s$ and $W^{\prime}$ above $M$ and hence above $W$. In this case $M \cap W=M^{\prime} \cap W$, so that membership of $M \cap W$ in $r$ reduces to membership of $M^{\prime} \cap W$ in $r$, for a node $M^{\prime}$ of $s$. Thus it is enough to consider the case when $M$ is a node of $s$. If there are transitive nodes $W^{\prime}$ of $s$ between $W$ and $M$, we may replace $M$ with $M \cap W^{\prime}$, since 
$M \cap W^{\prime}$ is also a node of $s$, and $M \cap W=\left(M \cap W^{\prime}\right) \cap W$. So, in sum, we may assume that $W$ is a node of $t-s, M$ is a node of $s-t$, and there are no transitive nodes of $s$ between $W$ and $M$. One can check in this case that $M$ belongs to $E_{W}$; hence $M \cap W$ belongs to $F_{W}$ and is a node of $r$ by definition.

Since the added intervals $F_{W}$ consist only of nodes that are intersections of nodes in $s$ with nodes in $t$, it follows from Claim 2.26 that $r$ is exactly equal to the closure of $s \cup t$ under intersections. To complete the proof of the lemma it remains to verify condition (3) of Definition 2.4 for $r$. The condition holds automatically at nodes of transitive type. For a small node $N$ of $r$ that belongs to $t$, we have $N \subseteq Q$, and therefore by Remark 2.24, $\{M \in r \mid M \in N\}=\{M \in r \mid M \in N \wedge M \in t\}$. Condition (3) for $r$ at $N$ therefore follows from the same condition for $t$. We must check the condition for the other small nodes of $r$, namely small nodes that belong to $s-t$, and small nodes in the added intervals $F_{W}$.

Claim 2.27 Let $N$ be a small node of $s$. Then every node of $r$ that belongs to $N$ is either a node of $s$ in $N$, or a node of $t$ in $N$, or the intersection of a small node of $s$ in $N$ with a transitive node of $t$ in $N$.

Proof Suppose not. Since the only nodes of $r$ that do not belong to $s \cup t$ are nodes in added intervals $F_{W}$, this means that there is a transitive node $W$ of $t$ and a node $M \in F_{W}$, equal to $M^{*} \cap W$, say, with $M^{*} \in E_{W}$, so that $M=M^{*} \cap W \in N$, but $M^{*}$ and $W$ do not both belong to $N$.

Suppose for simplicity that there is a transitive node of $s$ in the interval $(W, Q)$ of $s \cup t$, and let $W^{*}$ be the least such. The case when there are no transitive nodes of $s$ in that interval is similar.

Replacing $N$ with $N \cap W^{*}$ if needed, we may assume that $N$ occurs before $W^{*}$ in $s$. $N$ cannot occur at or before $M$ in $r$, since $M \in N$. And it cannot occur in the interval $(M, W)$ of $r$, since this interval is contained in $F_{W}$ which is disjoint from $s \cup t$. So $N$ must occur above $W$ in $r$. Thus, both $M^{*}$ and $N$ occur between $W$ and $W^{*}$ in $r$, and by minimality of $W^{*}$ it follows that there are no transitive nodes of $s$ between them.

If $N$ occurs before $M^{*}$, then by Claim 2.10, $N \in M^{*}$. Then $N \cap W \in M^{*} \cap W$ by a simple calculation using the fact that $W \in M^{*}$. On the other hand $M^{*} \cap W \in N$, and this implies that $M^{*} \cap W \in N \cap W$. Altogether then $N \cap W \in M^{*} \cap W \in N \cap W$, a contradiction. A similar argument leads to a contradiction in the case $N=M^{*}$.

So it must be that $N$ occurs above $M^{*}$. By Claim 2.10 it follows that $M^{*} \in N$ and that $M^{*} \subseteq N$. Since $W \in M^{*}$ it follows further that $W \in N$.

Claim 2.28 Let $N$ be a small node of s. Then $\{M \in r \mid M \in N\}$ belongs to $N$.

Proof By Claim 2.27, the closure of $r \supseteq s \cup t$ under intersections, and the elementarity of $N,\{M \in r \mid M \in N\}$ consists precisely of the nodes of $s$ that belong to $N$, the nodes of $t$ that belong to $N$, and all intersections of these nodes. Thus it is enough to prove that both $\{M \in s \mid M \in N\}$ and $\{M \in t \mid M \in N\}$ belong to $N .\{M \in r \mid M \in N\}$ is the closure of the union of these two sequences under intersections, and it belongs to $N$ by elementarity of $N$.

That $\{M \in s \mid M \in N\}$ belongs to $N$ is clear by condition (3) of Definition 2.4 for $s$. We prove that $\{M \in t \mid M \in N\}$ belong to $N$.

We can assume that there are no transitive nodes of $s$ above $N$ : otherwise, letting $W$ be the first such node, we can replace $s, Q$, and $t$ by $s \cap W, Q \cap W$, and $t \cap W$, 
respectively. We can also assume that there are no transitive nodes of $s$ above $Q$ : otherwise, letting $W$ be the first such node, we can replace $s$ and $N$ by $s \cap W$ and $N \cap W$, respectively.

We now divide into two cases. If $N$ occurs at or above $Q$, then (using Claim 2.10 and the assumptions in the previous paragraph) $Q \subseteq N$, and since $t \subseteq Q$ it follows that $\{M \in t \mid M \in N\}=t \in Q \subseteq N$. If $N$ occurs below $Q$, then (again using Claim 2.10 and the assumptions in the previous paragraph) $N \in Q$, and since $t \supseteq \operatorname{res}_{Q}(s)$ it follows that $N \in t$; hence $\{M \in t \mid M \in N\} \in N$ by condition (3) of Definition 2.4 for $t$.

Claim 2.29 Let $N$ be a small node of $r$ that belongs to an added interval $F_{W}$. Then $\{M \in r \mid M \in N\}$ belongs to $N$.

Proof Let $N^{*} \in E_{W}$ be such that $N=N^{*} \cap W$. Then by Claim 2.28 $\left\{M \in r \mid M \in N^{*}\right\}$ belongs to $N^{*}$. It is shown in the paragraphs defining $E_{W}$ that $W \in N^{*}$. Thus by elementarity of $N^{*},\left\{M \in r \mid M \in N^{*}\right\} \cap W$ belongs to $N^{*}$. By closure of $W$ (condition (4) in Definition 2.2), $\left\{M \in r \mid M \in N^{*}\right\} \cap W$ belongs also to $W$. Thus $\{M \in r \mid M \in N\}=\left\{M \in r \mid M \in N^{*} \cap W\right\}=\left\{M \in r \mid M \in N^{*}\right\} \cap$ $W \in N^{*} \cap W=N$.

The last two claims complete the proof of condition (3) of Definition 2.4 for $r$ and with it the proof that $r$ is a condition. Since $r \supseteq s \cup t$, and all nodes of $r$ are in the closure of $s \cup t$ under intersections, this completes the proof of Lemma 2.21.

Remark 2.30 In the case when $\kappa=\omega$ and $\lambda=\omega_{1}$, condition (3) of Definition 2.4 holds trivially, and therefore Claims 2.27 through 2.29 in the proof of Lemma 2.21 are not necessary.

Corollary 2.31 Let $s$ be a condition, and let $Q$ be a node of $s$. Suppose that $t$ is a condition that belongs to $Q$ and extends $\operatorname{res}_{Q}(s)$. Then we have the following.

(1) $s$ and t are directly compatible.

(2) Let $r$ witness that $s$ and $t$ are directly compatible, meaning that $r$ is the closure of $s \cup t$ under intersections. Then $\operatorname{res}_{Q}(r)=t$.

(3) The small nodes of $r$ outside $Q$ are all of the form $N$ or $N \cap W$, where $N$ is a small node of $s$ and $W$ is a transitive node of $t$.

Proof Condition (1) is immediate from Lemma 2.20 if $Q$ is of transitive type and from Lemma 2.21 if $Q$ is of small type.

If $Q$ is of transitive type, then Lemma 2.20 shows that $r$ is simply $s \cup t$, and since $t$ extends $\operatorname{res}_{Q}(s)$ it is clear that $\operatorname{res}_{Q}(r)=t$.

If $Q$ is of small type, then by Remark 2.24, every node of $r$ that belongs to $Q$ is a node of $t$; in other words, $\operatorname{res} Q(r) \subseteq t$. Since $t \subseteq r$ and $t \subseteq Q$ the reverse inclusion holds trivially, completing the proof of condition (2).

Condition (3) is clear if $Q$ is transitive, since then all nodes of $r$ outside $Q$ are nodes of $s$. If $Q$ is small, then $r$ consists of $s \cup t$ together with the added intervals $F_{W}$ from the proof of Lemma 2.21. Nodes in $t$ belong to $Q$, and nodes in the added intervals are of the form $N \cap W$, for small $N \in s$ and transitive $W \in t$, by the definition of the added intervals.

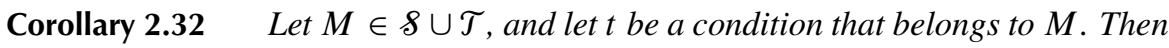
there is a condition $r \leq t$ with $M \in r$. Moreover, $r$ can be taken to be the closure of $t \cup\{M\}$ under intersections. 
Proof Let $s=\{M\}$. It is clear that $s$ is a condition and that $\operatorname{res}_{M}(s)=\emptyset$. Since $t \leq \emptyset$, by Corollary 2.31 the conditions $s$ and $t$ are directly compatible. Let $r$ witness this. Then $r \leq t, M \in r$, and $r$ is the closure of $t \cup\{M\}$ under intersections.

Claim 2.33 Let $s$ and $t$ be conditions, and let $W$ be a transitive node that belongs to both. Suppose that $s$ and $t$ are directly compatible and let $r$ witness this. Then $\operatorname{res}_{W}(r)$ is the closure of $\operatorname{res}_{W}(s) \cup \operatorname{res}_{W}(t)$ under intersections.

Proof Since $^{\operatorname{res}} W(s) \cup \operatorname{res}_{W}(t) \subseteq(s \cup t) \cap W$, the closure of $\operatorname{res}_{W}(s) \cup \operatorname{res}_{W}(t)$ under intersections is contained in $r$, contained in $W$, and hence contained in $\operatorname{res}_{W}(r)$.

Every node $M$ in $r$ is of the form $\bigcap_{i<k} M_{i}$ where $M_{i} \in s \cup t$. If $M$ belongs to $W$, then $M=M \cap W=\bigcap_{i<k} M_{i} \cap W$. By closure of $s$ and $t$ under intersections, and since $W$ belongs to both $s$ and $t, M_{i} \cap W \in s \cup t$. Since $M_{i} \cap W \in W$ or $M_{i} \cap W=W$, this implies that $M_{i} \cap W \in \operatorname{res}_{W}(s) \cup \operatorname{res}_{W}(t) \cup\{W\}$. Components $M_{i} \cap W$ which are equal to $W$ can be dropped from the intersection $\bigcap_{i<k} M_{i} \cap W$

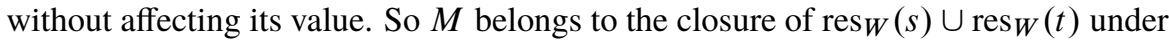
intersections.

We end this section with a slight modification of the poset $\mathbb{P}=\mathbb{P}_{\kappa, \&, \mathcal{T}, K}$, which we call the decorated version. We prove that this modified version satisfies a parallel of Corollary 2.31.

Claim 2.34 Let $s$ be a condition, and let $M$ and $N$ be nodes of $s$ so that $M \in N$. Let $M^{*}$ be the successor of $M$ in $s$. Then $M^{*} \subseteq N$.

Proof If $N$ is of transitive type, or there are no nodes of transitive type between $M$ and $N$, then $M^{*} \subseteq N$ by Claim 2.10. Suppose that $N$ is of small type and there are nodes of transitive type between $M$ and $N$. Let $W$ be the least one. By Claim 2.10, $M \in W$. Since $M \in N$, it follows that $M \in N \cap W$; hence in particular $N \cap W$ occurs above $M$. Since $N \cap W$ occurs below $W$, there are no nodes of transitive type between $M$ and $N \cap W$. By Claim 2.10, it follows that $M^{*} \subseteq N \cap W$.

Definition 2.35 Define $\mathbb{P}^{\text {dec }}=\mathbb{P}_{\kappa, \mathcal{S}, \mathcal{T}, K}^{\text {dec }}$ to be the poset consisting of pairs $\langle s, f\rangle$ where

(1) $s \in \mathbb{P}_{\kappa, 8, \mathcal{T}, K}$

(2) $f$ is a function on the nodes of $s$; for every $M \in s, f(M)$ is a set of size $<\kappa$

(3) if $M \in s$ is not the largest node in $s$, then $f(M)$ is an element of the successor of $M$ in $s$; if $M$ is the largest node of $s$, then $f(M) \in K$;

(4) $f$ belongs to $K$, and for every node $N \in s$, the restriction of $f$ to $\operatorname{res}_{N}(s)$ belongs to $N$.

Note that condition (4) holds automatically for transitive $N$; it follows for such $N$ using the closure of $N$ given by Definition 2.2.

The ordering on $\mathbb{P}^{\text {dec }}$ is the following: $\left\langle s^{*}, f^{*}\right\rangle \leq\langle s, f\rangle$ iff $s^{*} \leq s$, and $f^{*}(M) \supseteq f(M)$ for every $M \in s$.

Remark 2.36 In the case $\kappa=\omega$, condition (3) is equivalent to the requirement that $f(M) \subseteq M^{*}$ when $M^{*}$ is the successor of $M$ in $s$, and $f(M) \subseteq K$ when $M$ is the largest node of $s$. Moreover, condition (4) is automatically true in this 
case. By Claim 2.34, $f(M) \subseteq N$ for every $M \in \operatorname{res}_{N}(s)$, and together with the fact that $\operatorname{res}_{N}(s)$ and $f(M)$ for $M \in \operatorname{res}_{N}(s)$ are all finite, this automatically gives $f \uparrow \operatorname{res}_{N}(s) \in N$. Finiteness also automatically gives $f \in K$.

Thus, in the case $\kappa=\omega$, the definition simplifies to the following: $s \in \mathbb{P} ; f$ is a function defined on the nodes of $s$; and for each $M \in s, f(M)$ is a finite subset of the successor of $M$ in $s$ if there is one and of $K$ if $M$ is the largest node.

Let $\langle s, f\rangle \in \mathbb{P}^{\text {dec }}$, and let $Q$ be a node of $s$. Then the residue of $\langle s, f\rangle$ in $Q$, denoted $\operatorname{res}_{Q}(s, f)$, is defined to be $\left\langle\operatorname{res}_{Q}(s), f \uparrow \operatorname{res}_{Q}(s)\right\rangle$.

Claim 2.37 The residue $\operatorname{res}_{Q}(s, f)$ is a condition in $\mathbb{P}^{\mathrm{dec}}$ and belongs to $Q$.

Proof Conditions (1) and (2) of Definition 2.35 for $\operatorname{res}_{Q}(s, f)$ are clear from the same conditions for $\langle s, f\rangle$. Since $\operatorname{res}_{Q}(s) \in Q$, condition (4) for $\langle s, f\rangle$, used with $N=Q$, directly implies that $\operatorname{res}_{Q}(s, f)$ belongs to $Q$. Condition (4) transfers from $\langle s, f\rangle$ to $\operatorname{res}_{Q}(s, f): f \uparrow \operatorname{res}_{Q}(s)$ belongs to $K$ since it belongs to $Q$, and for any small node $N \in \operatorname{res} Q(s), f \uparrow \operatorname{res}_{Q}(s) \uparrow \operatorname{res}_{N}\left(\operatorname{res}_{Q}(s)\right)=f \uparrow \operatorname{res}_{N}(s) \in N$, using the fact that $N \subseteq Q$.

Finally, condition (3) for $\operatorname{res} Q(s, f)$ is immediate from the same condition for $s$, in all instances except when the successor of $M$ in $s$ is the bottom node of a residue gap of $s$ in $Q$. Let $[Q \cap W, W$ ) be the gap. By condition (3) for $s, f(M) \in Q \cap W$. In particular, $f(M) \in W$. Since $W$ is the successor of $M$ in res $Q(s)$, this establishes the $M$-instance of condition (3) for $\operatorname{res}_{Q}(s, f)$.

Two conditions $\langle s, f\rangle,\langle t, g\rangle \in \mathbb{P}^{\mathrm{dec}}$ are directly compatible in $\mathbb{P}^{\mathrm{dec}}$ if there is $\langle r, h\rangle$ witnessing their compatibility, with all nodes of $r$ obtained by intersections from nodes of $s \cup t$.

Claim 2.38 Let $\langle s, f\rangle \in \mathbb{P}^{\mathrm{dec}}$, let $Q$ be a node of $s$, and let $\langle t, g\rangle \in \mathbb{P}^{\mathrm{dec}}$ be an element of $Q$ that extends $\operatorname{res}_{Q}(s, f)$. Then $\langle s, f\rangle$ and $\langle t, g\rangle$ are directly compatible in $\mathbb{P}^{\mathrm{dec}}$.

Proof By Corollary 2.31, $s$ and $t$ are directly compatible in $\mathbb{P}$. Let $r$ witness this. It is enough now to find $h$ such that $\langle r, h\rangle \in \mathbb{P}^{\text {dec}}$; for every $M \in s, h(M) \supseteq f(M)$; and for every $M \in t, h(M) \supseteq g(M)$.

Set $h(M)$ to be equal to $g(M)$ for $M \in t$, equal to $f(M)$ for $M \in s-t$, and equal to $\emptyset$ for $M \in r-(s \cup t)$. Since $\langle t, g\rangle \leq \operatorname{res}_{Q}(s, f), g(M) \supseteq f(M)$ for $M \in s \cap t$. It follows that $h(M) \supseteq f(M)$ for $M \in s$. By definition, $h(M) \supseteq g(M)$ for $M \in t$. It remains to check that $\langle r, h\rangle$ satisfies the conditions in Definition 2.35.

Conditions (1) and (2) are clear. Fix $M \in r$ for condition (3). If $M$ is the largest node of $r$, then $M$ is the largest node of $s$, and $h(M)=f(M) \in K$. If $M \in s-t$, then $M$ is either at or above $Q$ or it is in a residue gap [ $Q \cap W, W$ ) of $s$ in $Q$. Either way the successor $M^{*}$ of $M$ in $r$ is the successor of $M$ in $s$, and $h(M)=f(M) \in M^{*}$. If $M \in r-(s \cup t)$, then $h(M)=\emptyset$, which certainly belongs to $M^{*}$. It remains to consider $M \in t$. If $Q$ is of transitive type, then $t$ is an initial segment of $r$, and $M^{*}$ is either the successor of $M$ in $t$ or $M^{*}=Q$. We have $h(M)=g(M) \in M^{*}$ by condition (3) for $\langle t, g\rangle$ in the former case, and since $\langle t, g\rangle \in Q$ in the latter. Suppose that $Q$ is of small type. If the successor $M^{*}$ of $M$ in $r$ is equal to the successor of $M$ in $t$, or to $Q$, then $h(M) \in M^{*}$ as in the case of transitive-type $Q$. If $M^{*}$ is the bottom node of a residue gap $[Q \cap W, W$ ) of $s$ in $Q$, then condition (3) for $\langle t, g\rangle$ yields $g(M) \in W$. Since 
$\langle t, g\rangle \in Q$, certainly $g(M) \in Q$. So $h(M)=g(M) \in Q \cap W=M^{*}$. By the proof of Lemma 2.21, the only other option is that $M^{*}$ is the lowest node of an added interval $F_{W}$ (in the terminology of the lemma). This lowest node is equal to $Q \cap W$, where $W$ is a transitive node of $t$ above $M$. Then by Claim 2.10 and condition (3) for $\langle t, g\rangle, g(M) \in W$. Furthermore, $g(M)$ belongs to $Q$ since $\langle t, g\rangle \in Q$. So $h(M)=g(M) \in Q \cap W=M^{*}$.

For condition (4), it is enough to prove that for small $N \in r, g \uparrow N$ belongs to $N$, and if $N \nsubseteq Q$, then $f \uparrow N$ belongs to $N$ too. This allows construction of $h \uparrow N$ inside $N$. (The full function $h$ can be constructed inside $K$ from $f$ and $g$.) Consider first the case when $N \in Q$. Then $N \subseteq Q$ because $N$ is small, and $N \in t$ by part (2) of Corollary 2.31. By condition (4) for $\langle t, g\rangle, g \uparrow N \in N$, as required.

Suppose next that $N \notin Q$. We prove that $f \uparrow N$ and $g \uparrow N$ both belong to $N$. The proof in both cases is by induction on $N$.

If $N$ is above $Q$, then $N \in s$, and this immediately implies $f \uparrow N \in N$. If there are no nodes of transitive type between $Q$ and $N$, then $Q \subseteq N$, and since $\langle t, g\rangle \in Q$ it follows that $g \uparrow N=g \in N$. If there are transitive nodes between $Q$ and $N$, let $R$ be such a node. By induction $g \uparrow N \cap R \in N \cap R \subseteq N$. Since $R$ is above $Q$ and $g \in Q, g \uparrow N \cap R=g \uparrow N$, so $g \uparrow N \in N$.

A similar argument applies if $N$ belongs to a residue gap of $s$ in $Q$, say, [ $Q \cap W, W$ ), using the fact that $N$ is above $Q \cap W$, and $g \uparrow N=g \uparrow Q \cap W$ in the case $Q \cap W \subseteq N$.

The only remaining alternative is that $Q$ is of small type and $N$ belongs to an added interval $F_{W}$ in the terminology of Lemma 2.21. In this case $N$ contains the smallest node of the interval, $Q \cap W$, and $g \uparrow N=g \uparrow Q \cap W$. Since $g \subseteq Q$, $g \uparrow Q \cap W=g \uparrow W$, which belongs to $W$ by condition (4) for $\langle t, g\rangle$ and to $Q$ since $g, W \in Q$. So $g \uparrow N=g \uparrow Q \cap W \in Q \cap W \subseteq N$. $N$ itself is equal to $N^{*} \cap W$ for some $N^{*} \in s$. By condition (4) for $\langle s, f\rangle, f \uparrow N^{*} \in N^{*}$. Since $N=N^{*} \cap W$, $f \uparrow N$ is an initial segment of $f \uparrow N^{*}$; hence it too belongs to $N^{*}$. It belongs to $W$ by closure of $W$ under sequences of length $<\kappa$. So $f \uparrow N \in N^{*} \cap W=N$.

Remark 2.39 As usual, the part of the proof of Claim 2.38 handling condition (4) of Definition 2.35 is not necessary in the case $\kappa=\omega$.

Corollary 2.40 Let $M \in \delta \cup \mathcal{T}$, and let $\langle t, g\rangle \in \mathbb{P}^{\text {dec }}$ be an element of $M$. Then there is $\langle r, h\rangle \in \mathbb{P}^{\mathrm{dec}}$ extending $\langle t, g\rangle$, with $M \in r$.

Proof The proof of this, from Claim 2.38, is similar to the proof of Corollary 2.32 from Corollary 2.31.

\section{Strong Properness}

This section includes some basic results about strong properness. The notion and the results presented are due to Mitchell [6], except for Claim 3.8, which is due to Friedman [3]. We also include some well-known results about properness and preservation of cardinals.

A condition $p$ in a poset $\mathbb{Q}$ is a strong master condition for a model $M$ if it forces $\dot{G} \cap M$ to be generic for $\mathbb{Q} \cap M$. In other words, it forces the generic filter to meet every dense subset of $\mathbb{Q} \cap M$. The poset is strongly proper for $M$ if every condition in $M$ can be extended to a strong master condition for $M$. 
Recall that $p$ is an ordinary master condition for $M$ if it forces the generic object to meet, inside $M$, every dense set of $\mathbb{Q}$ that belongs to $M$. $\mathbb{Q}$ is proper for $M$ if every condition in $M$ can be extended to a master condition for $M$.

Remark 3.1 If $p$ is a strong master condition for $M, \mathbb{Q} \in M$, and $M$ is sufficiently elementary in a transitive model, then $p$ is also an ordinary master condition for $M$. To see this note that for any dense set $D$ of $\mathbb{Q}$ that belongs to $M$, by elementarity $D \cap M$ is dense in $\mathbb{Q} \cap M$.

Remark 3.2 Suppose that $\mathbb{Q} \subseteq K$ is strongly proper for $M \subseteq K$. Let $\theta^{*}$ be large enough that $K \subseteq H\left(\theta^{*}\right)$, and suppose that $M^{*} \prec H\left(\theta^{*}\right)$ is such that $M^{*} \cap K=M$. Then $\mathbb{Q}$ is also strongly proper for $M^{*}$. This is immediate from the definitions, as only $M^{*} \cap \mathbb{Q}=M \cap \mathbb{Q}$ is relevant for determining strong properness.

The following claim gives standard consequences of properness. By the observation above, they are also consequences of strong properness.

Claim 3.3 Suppose that $M$ is elementary in $H\left(\theta^{*}\right)$ and that $\mathbb{Q} \in M$. Let $G$ be generic for $\mathbb{Q}$ over $V$, and suppose that $G$ includes a master condition for $M$. Then

(1) $M[G] \prec H\left(\theta^{*}\right)[G]$ and $M[G] \cap V=M$;

(2) let $\dot{f} \in M$, and suppose that $\dot{f}[G]$ is a function with ordinal domain; let $\tau=\dot{f} \cap M$; then $\tau[G]=\dot{f}[G] \uparrow M$.

Proof The first part is well known. For the second, it is clear that $\tau[G] \subseteq \dot{f}[G]$ and (using the first part) that $\operatorname{dom}(\tau[G]) \subseteq M$. For the reverse inclusion, if $\alpha \in M$ and $\dot{f}[G](\alpha)=b$, then there is some pair $\langle\sigma, q\rangle \in \dot{f}$ such that $\sigma[G]=\left\langle\alpha, b^{\prime}\right\rangle$, $q \in G$, and moreover, for every such pair, $b^{\prime}=b$. Using the first condition, such a pair $\langle\sigma, q\rangle$ can be found in $M$, so that it belongs to $\tau$.

Call a poset proper for $\delta$ if it is proper for every $M \in \mathcal{S}$, and similarly with strong properness.

Recall that $X \subseteq \mathcal{P}(Y)$ is stationary in $Y$ if it meets every club subset of $\mathcal{P}(Y)$ or, more precisely, if for every function $f: Y^{<\omega} \rightarrow Y$, there is $u \in X$ which is closed under $f$.

Claim 3.4 Suppose that $\mathbb{Q} \in H\left(\theta^{*}\right)$ is proper for $\AA^{*}$. Let $\delta<\theta^{*}$ be a cardinal, and suppose that for each $\alpha<\delta$, the set $\left\{M \in \delta^{*} \mid \alpha \subseteq M\right.$ and $\left.|M|<\delta\right\}$ is stationary in $H\left(\theta^{*}\right)$. Then forcing with $\mathbb{Q}$ does not collapse $\delta$.

Proof This is a standard application of Claim 3.3. Let $\dot{f}$ be a name for a function into $\delta$, with domain $\alpha<\delta$. Let $p \in \mathbb{Q}$. Using the stationarity assumed in the claim, find $M \in \delta^{*}$ of size $<\delta$ with $\alpha \cup\{\dot{f}, p, \mathbb{Q}\} \subseteq M \prec H\left(\theta^{*}\right)$. Let $q \leq p$ be a master condition for $M$. By Claim 3.3, $q$ forces the range of $\dot{f}$ to be contained in $M$. Since $M \in V$ has size $<\delta, M \nsupseteq \delta$, and hence $q$ forces $\dot{f}$ to not be onto $\delta$.

Claim 3.5 Suppose that $\mathbb{Q} \subseteq K$ is strongly proper for 8 . Let $\delta$ be a cardinal, and suppose that for each $\alpha<\delta$, the set $\{M \in \delta \mid \alpha \subseteq M$ and $|M|<\delta\}$ is stationary in $K$. Then forcing with $\mathbb{Q}$ does not collapse $\delta$.

Proof Let $\theta^{*}$ be large enough that $K, \mathbb{Q} \in H\left(\theta^{*}\right)$. Let $8^{*}=\left\{M^{*} \subseteq H\left(\theta^{*}\right) \mid\right.$ $\left.M^{*} \cap K \in \delta\right\}$. Then the stationarity assumed in the claim implies that $\left\{M \in 8^{*} \mid\right.$ $\alpha \subseteq M$ and $|M|<\delta\}$ is stationary in $H\left(\theta^{*}\right)$. Moreover, $\mathbb{Q}$ is strongly proper for 
each $M^{*} \in \boldsymbol{S}^{*}$ by Remark 3.2 and hence is proper for $M^{*}$ by Remark 3.1. The current claim now follows from Claim 3.4.

Lemma 3.6 Let $G$ be generic for $\mathbb{Q}$ over $V$, let $\alpha$ be an ordinal, and let $f=\dot{f}[G] \in V[G]$ be a function from $\alpha$ into the ordinals. Suppose that $\dot{f}, \mathbb{Q} \in M$, $M$ is elementary in some $H\left(\theta^{*}\right)$, and $G$ includes a strong master condition for $M$. If $f \uparrow M$ belongs to $V$, then the entire function $f$ must belong to $V$.

Proof Redefining the name $\dot{f}$ if necessary, we may assume that all elements of $\dot{f}$ are of the form $\left\langle\langle\xi, \mu\rangle^{\nu}, t\right\rangle$, where $\xi<\alpha, \mu \in$ Ord, and $t \Vdash \dot{f}(\breve{\xi})=\check{\mu}$. Let $\tau=\dot{f} \cap M$. By Claim 3.3 and Remark 3.1, $\tau[G]=\dot{f}[G] \uparrow M$. Suppose that $\tau[G]$ belongs to $V$. We prove that $f$ belongs to $V$.

We are assuming that $G$ includes a strong master condition for $M$, and hence $G \cap M$ is generic for $\mathbb{Q} \cap M$ over $V$. Since $\tau \subseteq M$ and all elements of $\tau$ are of the form $\left\langle\langle\xi, \mu\rangle^{\nu}, t\right\rangle, \tau$ is a $\mathbb{Q} \cap M$-name. $\tau[G]$ (with $\tau$ viewed as a $\mathbb{Q}$-name) is equal to $\tau[G \cap M]$ (with $\tau$ viewed as a $(\mathbb{Q} \cap M)$-name). Since $\tau[G \cap M]=\tau[G]$ belongs to $V$, there is a condition $r \in G \cap M$ which forces (in $\mathbb{Q} \cap M$ ) a specific value for $\tau$. In particular, for every $\xi \in \alpha \cap M$, there is $\mu \in \operatorname{Ord} \cap M$ such that every $s \in \mathbb{Q} \cap M$ with $s \leq r$ extends to $t \in \mathbb{Q} \cap M$ so that $\left\langle\langle\xi, \mu\rangle^{2}, t\right\rangle \in \dot{f}$. By elementarity of $M$, it follows that for every $\xi \in \alpha$, there is $\mu \in \operatorname{Ord}$ such that every $s \in \mathbb{Q}$ with $s \leq r$ extends to $t \in \mathbb{Q}$ so that $\left\langle\langle\xi, \mu\rangle^{\nu}, t\right\rangle \in \dot{f}$. This implies that $r$ in fact completely forces, in $\mathbb{Q}$, all values of $\dot{f}$. So $f=\dot{f}[G] \in V$.

Lemma 3.7 $\quad$ Suppose that $\mathbb{Q} \subseteq K$ is strongly proper for 8 , let $\delta$ be a regular cardinal, and suppose that $\{M \in \delta|| M \mid<\delta\}$ is stationary in $K$. Then forcing with $\mathbb{Q}$ does not add branches of length $\delta$ to trees in $V$. Precisely, suppose that $T$ is a tree in $V, G$ is generic for $\mathbb{Q}$ over $V$, and $b \in V[G]$ is a branch through $T$ of length $\delta$. Then $b \in V$.

Proof Without loss of generality we may assume that nodes of $T$ are ordinals, so that branches of length $\delta$ through $T$ are functions from $\delta$ into ordinals. Suppose for contradiction that $b=\dot{b}[G]$ is a branch through $T$, of length $\delta$, that belongs to $V[G]$ but not to $V$. Let $r \in G$ be a condition forcing this.

Let $\theta^{*}$ be large enough that $K, \mathbb{Q}, \dot{b} \in H\left(\theta^{*}\right)$. Using the stationarity assumed in the lemma, fix $M^{*} \prec H\left(\theta^{*}\right)$ so that $\left|M^{*}\right|<\delta, r, \dot{b}$, and $\mathbb{Q}$ belong to $M^{*}$, and $M^{*} \cap K \in \delta$. Let $p \leq r$ be a strong master condition for $M^{*} \cap K$ (equivalently, since $\mathbb{Q} \subseteq K$, a strong master condition for $M^{*}$ ). Replacing the generic $G$ if needed, we may assume that $p \in G$.

Then since $b \notin V$ it follows by Lemma 3.6 that $b \uparrow M^{*} \notin V$. Let $\gamma=\sup (\delta \cap$ $\left.M^{*}\right)$. Since $\delta$ is regular and $\left|M^{*}\right|<\delta, \gamma$ is smaller than $\delta$. Since $T$ is a tree that belongs to $V$, this implies that $b \uparrow \gamma$ belongs to $V$. (It is the function that enumerates all $T$-predecessors of the node $b(\gamma)$ according to their order in $T$.) Since $b \uparrow M^{*}=b \uparrow \gamma \uparrow M^{*}$ it follows that $b \uparrow M^{*}$ belongs to $V$, a contradiction.

The next claim, which deals with the product of proper and strongly proper posets, is an abstraction of Friedman [3, Lemma 3].

Claim 3.8 Let $\mathbb{A}, \mathbb{P} \in M \prec H\left(\theta^{*}\right)$. Suppose that $\mathbb{A}$ is strongly proper for $M$ and that $\mathbb{P}$ is proper for $M$. Then 
(1) if $a$ and $p$ are strong and ordinary master conditions for $M$ in $\mathbb{A}$ and $\mathbb{P}$, respectively, then $\langle a, p\rangle$ is a master condition for $M$ in $\mathbb{A} \times \mathbb{P}$;

(2) $\mathbb{A} \times \mathbb{P}$ is proper for $M$;

(3) if $G$ is generic for $\mathbb{A}$ over $V$ with a strong master condition for $M$, then in $V[G], \mathbb{P}$ is proper for $M[G]$.

Proof We prove the first part of the claim. The other two parts are immediate consequences of the first. Let $D \in M$ be a dense subset of $\mathbb{A} \times \mathbb{P}$, and let $a$ and $p$ be strong and ordinary master conditions for $M$ in $\mathbb{A}$ and $\mathbb{P}$, respectively. It is enough to prove, for every such pair $\langle a, p\rangle$, that $\langle a, p\rangle$ is compatible with an element of $D \cap M$. Let $Z=\{b \in \mathbb{A} \cap M \mid(\exists q \in M)\langle b, q\rangle \in D$ and $q$ is compatible with $p\}$. The density of $D$, the elementarity of $M$, and the fact that $p$ is a master condition for $M$ in $\mathbb{P}$ imply that $Z$ is dense in $\mathbb{A} \cap M$. Since $a$ is a strong master condition for $M$ there is $b \in Z$ which is compatible with $a$. By definition of $Z$ there is then $q \in M$ such that $\langle b, q\rangle \in D$ and $q$ is compatible with $p$.

\section{Sequence Poset and Strong Properness}

Let $\delta$ and $\mathcal{T}$ be appropriate for $\kappa, \lambda$, and $K$. Let $\mathbb{P}$ be the sequence poset associated to $\kappa, \delta, \mathcal{T}$, and $K$.

\section{Claim 4.1}

(1) Let $s \in \mathbb{P}$, and let $Q$ be a node in $s$. Then $s$ is a strong master condition for $Q$.

(2) $\mathbb{P}$ is strongly proper for $\delta \cup \mathcal{T}$.

(3) If $W \in \delta \cup \mathcal{T}$, then $\mathbb{P} \cap W$ is strongly proper for $(\delta \cup \mathcal{T}) \cap W$. For any condition $s \in \mathbb{P} \cap W$ and any node $Q \in s, s$ is a strong master condition for $Q$ in $\mathbb{P} \cap W$.

Proof Consider first condition (1). Suppose for contradiction that $s$ is not a strong master condition for $Q$. Extending $s$, we may fix a dense subset $D$ of $\mathbb{P} \cap Q$ and assume that $s$ forces the generic filter for $\mathbb{P}$ to avoid $D$. Note that res $Q(s)$ is a condition of $\mathbb{P}$ that belongs to $Q$. By density of $D$, there is $t \in D$ extending $\operatorname{res}_{Q}(s)$. We have $t \in Q$ as $D \subseteq Q$. By Corollary 2.31, $s$ and $t$ are directly compatible. Let $r$ witness this. Then $r$ is an extension of $s$ that forces $t$ into the generic object, contradicting the fact that $s$ forces the generic object to avoid $D$.

By Corollary 2.32, every condition $u \in Q$ extends to a condition $s$ with $Q \in s$, which by (1) is a strong master condition for $\mathbb{P}$. This establishes condition (2).

For condition (3), note that if $s$ and $t$ in the proof of condition (1) both belong to $W$, then so does $r$, since by elementarity $W$ is closed under intersections. Similarly, in the proof of condition (2), if $u$ and $Q$ belong to $W$, then so does $s$. The same proofs can therefore be used to get the strong properness of $\mathbb{P} \cap W$.

Claim 4.2 Let $\mathbb{P}^{\text {dec }}$ be the decorated sequence poset associated to $\kappa, \lambda, 8$, and $\mathcal{T}$. Then $\mathbb{P}^{\mathrm{dec}}$ is strongly proper for $\delta \cup \mathcal{T}$. Moreover, any condition $\langle t, g\rangle \in \mathbb{P}^{\mathrm{dec}}$ that belongs to a node $Q \in \mathcal{S} \cup \mathcal{T}$ extends to a condition $\langle s, f\rangle \in \mathbb{P}^{\operatorname{dec}}$ with $Q \in s$, and any such condition $\langle s, f\rangle$ is a strong master condition for $Q$.

Proof This is similar to the proof of Claim 4.1 but uses Claim 2.38 and Corollary 2.40 instead of Corollaries 2.31 and 2.32. 
Let $W$ be a node. $\{W\}$ is then a strong master condition for $W$, meaning that if $G$ is generic for $\mathbb{P}$ over $V$ with $\{W\} \in G$, then $\bar{G}=G \cap W$ is generic for $\overline{\mathbb{P}}=\mathbb{P} \cap W$ over $V$. This implies that the forcing to add $G$ can be broken into two stages: first force with $\overline{\mathbb{P}}$ to add $\bar{G}$; then force with a factor poset to add $G$ over $V[\bar{G}]$. It is easy to check that the factor poset is simply the restriction of $\mathbb{P}$ to conditions $s$ so that $W \in S$ and $\operatorname{res}_{W}(s) \in \bar{G}$. (This poset belongs to $V[\bar{G}]$.) We continue with a few claims and remarks on strong properness for the factor poset.

Claim 4.3 Let $W$ be a transitive node, let $\overline{\mathbb{P}}=\mathbb{P} \cap W$, and let $\bar{G}$ be generic for $\overline{\mathbb{P}}$ over $V$. Let $\hat{\delta}=\{M \in \delta \mid W \in M$ and $M \cap W \in \bigcup \bar{G}\}$. Suppose that, in $V, s$ is stationary in $K$. Then, in $V[\bar{G}], \hat{\rho}$ is stationary in $K$.

Proof Suppose for contradiction that $f=\dot{f}[\bar{G}]: K^{<\omega} \rightarrow K$ and no element of $\hat{s}$ is closed under $f$. Let $\bar{s} \in \bar{G}$ force this.

Let $\theta^{*}$ be large enough that $K \in H\left(\theta^{*}\right)$, and using the stationarity of 8 , find

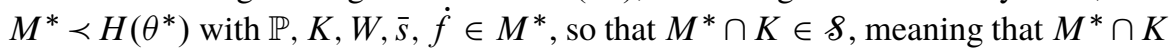
is a small node. Let $M$ denote $M^{*} \cap K$. Since $W \in M, M \cap W$ too is a small node and belongs to $W$. Let $\bar{r} \in \overline{\mathbb{P}}$ be an extension of $\bar{s}$ with $M \cap W \in \bar{r}$. Such an extension exists in $\mathbb{P}$ by Corollary 2.32, and by elementarity of $W$ it can be taken to belong to $W$ and hence to $\overline{\mathbb{P}}$. Changing $\bar{G}$ if needed, we may assume that $\bar{r} \in \bar{G}$. By Claim 4.1, $\bar{r}$ is a strong master condition for $M \cap W=M^{*} \cap W$ in $\overline{\mathbb{P}} \subseteq W$ and hence by Remark 3.2 also a strong master condition for $M^{*}$. By Remark 3.1 and Claim 3.3, $M^{*}[\bar{G}] \prec H\left(\theta^{*}\right)[\bar{G}]$ and $M^{*}[\bar{G}] \cap V=M^{*}$. Since $\dot{f} \in M^{*}$ it follows that $M^{*}$ is closed under $f$, and hence so is $M$. This is a contradiction since $\bar{r}$ forces $M$ into $\hat{\rho}$.

Claim 4.4 Let $W$ be a transitive node, let $\overline{\mathbb{P}}=\mathbb{P} \cap W$, let $\bar{G}$ be generic for $\overline{\mathbb{P}}$ over $V$, and let $\mathbb{Q}$ be the factor poset for adding a $V$ generic for $\mathbb{P}$ extending $\bar{G}$ over $V[\bar{G}]$. Let $\hat{\mathcal{S}}$ be defined as in Claim 4.3. Then $\mathbb{Q}$ is strongly proper for $\hat{\boldsymbol{s}}$.

Note that $M[\bar{G}] \cap V=M$ for any $M \in \hat{\delta}$, since $\{M \cap W\}$ is a strong master condition for $M$ in $\mathbb{P} \cap W$. Since $\mathbb{Q} \subseteq V$, and since the definition of a strong master condition for $M^{*}$ in $\mathbb{Q}$ depends only on $M^{*} \cap \mathbb{Q}$, it follows that for $M \in \hat{s}$, every strong master condition for $M$ in $\mathbb{Q}$ is also a strong master condition for $M[\bar{G}]$. Claim 4.4 therefore shows that $\mathbb{Q}$ is strongly proper for $\{M[\bar{G}] \mid M \in \hat{8}\}$.

Proof of Claim 4.4 Fix $M \in \delta$ with $W \in M$ and $M \cap W \in \bigcup \bar{G}$. We have to show that the factor poset $\mathbb{Q}$ is strongly proper for $M$. We show this by proving that (1) any condition $s \in \mathbb{Q}$ with $M \in s$ is a strong master condition for $M$ in $\mathbb{Q}$; and (2) any condition $t \in \mathbb{Q}$ that belongs to $M$ can be extended to such an $s$.

Consider (1) first. Suppose for contradiction that $s$ is not a strong master condition for $M$. Extending $s$ if necessary, we may assume that it forces that the generic avoids a specific $D \subseteq \mathbb{Q} \cap M$ which is dense in $\mathbb{Q} \cap M$. So no extension $r$ of $s$ in $\mathbb{Q}$ extends an element of $D$.

Recall that the factor poset $\mathbb{Q}$ consists of conditions $u \in \mathbb{P}$ so that $W \in u$ and $\operatorname{res}_{W}(u) \in \bar{G}$. The fact that $s$ is such a condition, $M \in s$, and $W \in M$, implies that so is $\operatorname{res}_{M}(s)$. (To see that $\operatorname{res}_{W}\left(\operatorname{res}_{M}(s)\right)$ belongs to $\bar{G}$, note that it is weaker than $\operatorname{res}_{W}(s)$, which belongs to $\bar{G}$.) By density of $D$, there is some $t \in D$ extending $\operatorname{res}_{M}(s)$. By Corollary 2.31 and since $D \subseteq M, s$ and $t$ are directly compatible in $\mathbb{P}$. To derive a contradiction we need to show they are compatible in the factor poset. 
Let $r$ witness that $s$ and $t$ are directly compatible in $\mathbb{P}$. By Claim 2.33, $\operatorname{res}_{W}(r)$ is the closure of $\operatorname{res}_{W}(s) \cup \operatorname{res}_{W}(t)$ under intersections. From this, the fact that $\operatorname{res}_{W}(s)$ and $\operatorname{res}_{W}(t)$ both belong to $\bar{G}$, and the fact that $\bar{G}$ is a filter, it follows that $\operatorname{res}_{W}(r)$ too belongs to $\bar{G}$. Hence $r$ belongs to the factor poset, witnessing that $s$ and $t$ are compatible in this poset. This completes the proof of (1).

Assertion (2) is a consequence of the proof of (1). Let $t$ be a condition in the factor poset that belongs to $M$. Let $u$ be the condition $\{M \cap W, W, M\}$. Then $u$ is a condition in the factor poset, $\operatorname{res}_{M}(u)=\{W\} \subseteq t$, and the proof of (1) shows that $u$ and $t$ are compatible in the factor poset. Any condition $s$ in the factor poset witnessing this is an extension of $t$ with $M \in s$.

Remark 4.5 We only proved strong properness of the factor poset for small nodes. Similar strong properness for transitive nodes is also true. To be more precise, let $\hat{\mathcal{T}}=\{M \in \mathcal{T} \mid W \in M\}$. It is easy to check that stationarity of $\mathcal{T}$ in $V$ implies stationarity of $\hat{\mathcal{T}}$ in $V[\bar{G}]$, and that the factor poset is strongly proper for $\hat{\mathcal{T}}$. The proofs are similar to the proofs of Claims 4.3 and 4.4 but simpler in several places.

Remark 4.6 We worked in Claims 4.3 and 4.4 under the assumption that $W$ is transitive. Similar claims hold for small $W$, and again the proofs are similar to the proofs of the claims but simpler. To be more precise, if $W$ is small, then the factor poset is strongly proper for $\hat{\delta}=\{M \in \mathcal{S} \mid W \in M\}$ and for $\hat{\mathcal{T}}=\{M \in \mathcal{T} \mid W \in M\}$, and each of these sets is stationary in $V[\bar{G}]$ if it is stationary in $V$.

\section{Initial Applications}

We can now give several quick applications of the two-type model sequence posets defined in Section 2. We use the posets to obtain the tree property, to add clubs in subsets of $\theta \geq \omega_{2}$, to collapse to $\kappa^{+}$without adding branches of length $\kappa^{+}$to trees in $V$, and to obtain a model of PFA with an inner model that is correct about $\omega_{2}$ but not about reals. The first two are the initial applications of the finite condition posets of countable models developed by Friedman [2] and Mitchell [6]. Our poset gives the same extensions, but the use of models of two types rather than only countable models makes the proof conceptually simpler. The last is a sketch of the argument of Friedman [3] but using the two-type model sequences.

5.1 The tree property (after Mitchell [6]) Let $\theta$ be a weakly compact cardinal. Let $K=H(\theta)$. Let $\mathcal{T}$ consist of all transitive $W \prec K$ of size $<\theta$ which are countably closed, and let $\delta$ consist of all countable $M \prec K$. Both $\delta$ and $\mathcal{T}$ are stationary. The

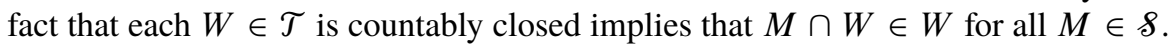
It is easy to check that if $W \in \mathcal{T}, M \in \mathcal{S}$, and $W \in M$, then $M \cap W \prec K$, and therefore $M \cap W \in \delta$. (By elementarity of $M$, and since $W$ can be well-ordered inside $K$, there is a well-ordering of $W$ in $M$. This allows defining Skolem functions for $W$, inside $M$. Using the Skolem functions and the elementarity of $M$ one can check that $M \cap W \prec W$, and this implies $M \cap W \prec K$.)

$\delta$ and $\mathcal{T}$ are therefore appropriate for $\omega, \omega_{1}$, and $K=H(\theta)$. Let $\mathbb{P}=\mathbb{P}_{\omega, \delta, \mathcal{T}, K}$. By Claim 4.1, $\mathbb{P}$ is strongly proper for every $Q \in \delta \cup \mathcal{T}$, and indeed every condition $s$ with $Q \in s$ is a strong master condition for $Q$. It follows by Claim 3.5 that forcing with $\mathbb{P}$ preserves $\omega_{1}$ and $\theta$, using the stationarity of $\delta$ for the former and the stationarity of $\mathcal{T}$ for the latter. 
Let $G$ be generic for $\mathbb{P}$ over $V$. Then $\bigcup G$ is a set of nodes. Using Claim 2.10, it is clear that $A=\{W \in \bigcup G \mid W$ is a transitive node $\}$ is increasing, both in $\in$ and in $\subseteq$. Moreover, by genericity and Corollary 2.32, $A$ is unbounded in $H(\theta)$, meaning that for every $x \in H(\theta)$, there is $W \in A$ with $x \in W$. In particular, this means that $\bigcup A=H(\theta)$.

For each $W \in A$, let $B_{W}=\{M \in \bigcup G \mid M$ is a small node between $W$ and $\left.W^{*}\right\}$, where $W^{*}$ is the next element of $A$ above $W$. Using Claim 2.10, it is clear that for every $W \in A, B_{W}$ is increasing, both in $\in$ and in $\subseteq$. By genericity and Corollary 2.32, for every $x \in H(\theta)$ there is a small node $M$ in $\bigcup G$ with $\{W, x\} \subseteq M$. Since all conditions in $G$ are closed under intersection and $W^{*} \in \bigcup G, M \cap W^{*}$ too belongs to $\bigcup G$ and must occur between $W$ and $W^{*}$ since $W \in M \cap W^{*}$. Letting $x$ range over elements of $W^{*}$, it follows that $\bigcup B_{W}=W^{*}$.

Since $B_{W}$ is increasing and all models in $B_{W}$ are countable, the length of $B_{W}$ is at most $\omega_{1}^{V}$. Again using the fact that all models in $B_{W}$ are countable, it follows that $W^{*}=\bigcup B_{W}$ has size at most $\omega_{1}^{V}=\omega_{1}^{V[G]}$ in $V[G]$.

Since this is true for each $W \in A$, and since every ordinal below $\theta$ belongs to some $W \in A$, it follows that in $V[G]$, every ordinal between $\omega_{1}$ and $\theta$ is collapsed to $\omega_{1}$. From this and the preservation of $\theta$, it follows that $\omega_{2}^{V[G]}=\theta$. We continue to prove that in $V[G]$, the tree property holds at $\theta$.

Claim 5.1 In $V[G]$, the tree property holds at $\theta=\omega_{2}$.

Proof Suppose not, and let $\dot{T} \in V$ be a name for a tree witnessing this. We may assume that elements of $T=\dot{T}[G]$ are pairs $\langle\xi, \mu\rangle \in \theta \times \omega_{1}$ and that level $\xi$ of $T$ consists exactly of $\{\xi\} \times \omega_{1}$. We may also assume that $\dot{T} \subseteq H(\theta)$. Finally, we may assume for definitiveness that it is forced outright in $\mathbb{P}=\mathbb{P}_{\omega, \mathcal{S}, \mathcal{T}, H(\theta)}$ that there are no cofinal branches through $\dot{T}$.

By the $\Pi_{1}^{1}$-indescribability of weakly compact cardinals (see Jech [5, Theorem 17.18]), there is an inaccessible cardinal $\kappa<\theta$ such that

(1) $(H(\kappa) ; \dot{T} \cap H(\kappa)) \prec(H(\theta), \dot{T})$;

(2) it is forced in $\overline{\mathbb{P}}=\mathbb{P} \cap H(\kappa)$ that $\dot{T} \cap H(\kappa)$ has no branches of length $\kappa$;

(3) it is forced in $\overline{\mathbb{P}}$ that $\kappa$ is regular.

Let $W=H(\kappa) . W$ is then a node of transitive type, and changing $G$ if necessary, we may assume $\{W\} \in G$. By strong properness of $\mathbb{P}$ then, $\bar{G}=G \cap W$ is generic over $V$ for $\mathbb{P} \cap W$. By the conditions above, in $V[\bar{G}], \kappa$ is a regular cardinal, and $\bar{T}=(\dot{T} \cap W)[\bar{G}]$ is a tree on $\kappa \times \omega_{1}$, with no branches of length $\kappa$.

An application of Remark 3.1 and Claim 3.3 shows that $(H(\kappa) ; \bar{T}) \prec(H(\theta) ; T)$, and this implies that $\bar{T}$ is equal to $T \uparrow \kappa$. In particular any node on level $\kappa$ of $T$ determines a branch of length $\kappa$ in $\bar{T}$. Thus, in $V[G]$, there are branches of length $\kappa$ through $\bar{T}$.

Let $\mathbb{Q} \in V[\bar{G}]$ be the factor poset to add $G$, forcing over $V[\bar{G}]$. Let $\hat{\delta}$ be as in Claim 4.3. By the claim, $\hat{\delta}$ is a stationary set of countable elementary substructures of $H(\theta)$ in $V[\bar{G}]$. By Claim 4.4, $\mathbb{Q}$ is strongly proper for $\hat{\delta}$. It follows by Lemma 3.7 that forcing with $\mathbb{Q}$ over $V[\bar{G}]$ does not add any branches of length $\kappa$ to $\bar{T}$. But this is a contradiction, since $V[G]$ is an extension of $V[\bar{G}]$ by $\mathbb{Q}$, and $\bar{T}$, which has no branches of length $\kappa$ in $V[\bar{G}]$, has such branches in $V[G]$. 
By similar proofs, but with $\kappa>\omega$ (and $\lambda=\kappa^{+}$), one can of course obtain the tree property at greater cardinals. The sequence poset can also be used to obtain the tree property at two successive cardinals. For example, suppose that $\theta$ is supercompact and $\theta^{*}>\theta$ is weakly compact. Force with the poset $\mathbb{P}$ above to add $G$. Then follow this by forcing, over $V[G]$, with the sequence poset associated to $\omega_{1}, \theta=\omega_{2}$, with 8 consisting of models of the form $M[G]$ where $\theta \in M \prec H\left(\theta^{*}\right), M$ belongs to $V$, has size $<\theta$, has ordinal intersection with $\theta$, and $M \cap H(\theta) \in \bigcup G$, and with $\mathcal{T}$ consisting of models $W[G]$ where $W \prec H\left(\theta^{*}\right)$ is transitive and $\theta \in W$. This will collapse all ordinals between $\theta$ and $\theta^{*}$ to $\theta$, resulting in a model where $\theta^{*}=\omega_{3}$ and where the tree property holds at both $\theta$ and $\theta^{*}$.

However, the proof in this case is substantially more involved for a couple of reasons. The second-stage sequence poset forcing uses small nodes which are not countably closed in $V[G]$. While $\theta$ and $\theta^{*}$ are preserved by this forcing using strong properness, preservation of $\omega_{1}$ requires a special argument. And while the proof of the tree property at $\theta^{*}$ is identical to the proof given above using strong properness of the factor poset, the proof at $\theta$ is more involved since factors of the second-stage poset to its small nodes are not strongly proper after forcing with the first stage.

5.2 Adding clubs with finite conditions (after Friedman [2] and Mitchell [6]) Let $\theta \geq \omega_{2}$ be a regular cardinal. We show, under certain conditions which we will explain below, how to add club subsets to stationary subsets of $\theta$, using finite conditions.

Lemma 5.2 Let $K$ be a transitive set with $K \cap$ Ord $=\theta$, satisfying enough of ZFC for the properties listed at the beginning of Section 2. Let 8 and $\mathcal{T}$ be appropriate for $\omega, \omega_{1}$, and $K$. Let $\mathbb{P}^{\text {dec }}$ be the decorated sequence poset associated to $\omega$, $8, \mathcal{T}$, and $K$.

(1) If $\&$ and $\mathcal{T}$ are stationary in $K$, then $\mathbb{P}^{\mathrm{dec}}$ does not collapse $\omega_{1}$ and $\theta$.

(2) If $|K|=\theta$, then $\mathbb{P}^{\text {dec }}$ is $\theta^{+}$-c.c. and does not collapse cardinals above $\theta$.

(3) If $\delta \cup \mathcal{T}$ is unbounded in $K$, meaning that for every $x \in K$ there is

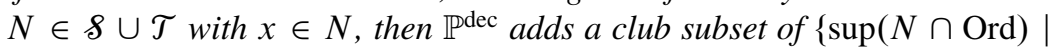

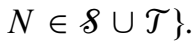

Proof The first part is immediate from the strong properness of $\mathbb{P}^{\text {dec }}$, given by Claim 4.2, using Claim 3.5. The second part is clear as $\mathbb{P}^{\text {dec }} \subseteq K$. For the third part, let $G$ be generic for $\mathbb{P}^{\text {dec }}$ over $V$, and let $C=\{\sup (N \cap$ Ord $) \mid N \in \cup G\}$. It is clear that $C \subseteq\{\sup (N \cap$ Ord $) \mid N \in \delta \cup \mathcal{T}\}$. We prove that $C$ is a club in $\theta$.

Using Corollary 2.40, the fact that $\delta \cup \mathcal{T}$ is unbounded in $K$ implies that for every $\alpha \in K$, the set of conditions forcing an ordinal above $\alpha$ into $C$ is dense. It follows that $C$ is unbounded in $\theta=K \cap$ Ord.

It remains to show that $C$ is closed. Let $\alpha<\theta$, and let $\langle s, f\rangle \in \mathbb{P}^{\text {dec }}$ force $\alpha \notin C$. It is enough to show that $\langle s, f\rangle$ can be extended to a condition forcing that $\alpha$ is not a limit point of $C$. Let $Q$ be the first node in $s$ so that $\sup (Q \cap$ Ord $) \geq \alpha$. (Extending $s$ if necessary we may assume that there is such a node, using the unboundedness of $C$.) Let $M$ be the largest node of $s$ below $Q$. Since $\langle s, f\rangle$ forces $\alpha$ outside $C$, $\sup (Q \cap$ Ord $)>\alpha$. Hence there is $\xi \geq \alpha$ which belongs to $Q$. Let $f^{\prime}$ be the function on $s$ that differs from $f$ only on $M$, with $f^{\prime}(M)=f(M) \cup\{\xi\}$. Then $\left\langle s, f^{\prime}\right\rangle \in \mathbb{P}^{\text {dec }}$ and $\left\langle s, f^{\prime}\right\rangle \leq\langle s, f\rangle$. Moreover, in every extension $\langle t, g\rangle$ of $\left\langle s, f^{\prime}\right\rangle$, the successor of $M$ is a node $M^{*}$ with $\xi \in M^{*}$, and hence $\sup \left(M^{*} \cap\right.$ Ord $)>\alpha$. It follows that 
$\left\langle s, f^{\prime}\right\rangle$ forces that $C$ has no elements between $\sup (M \cap$ Ord $)$ and $\alpha$, and in particular $\alpha$ is not a limit point of $C$.

By Lemma 5.2, adding a club subset of $U \subseteq \theta$ using finite conditions reduces to finding appropriate $\delta$ and $\mathcal{T}$ so that $\{\sup (N \cap$ Ord $) \mid N \in \delta \cup \mathcal{T}\} \subseteq U$. To ensure preservation of cardinals, $\delta$ and $\mathcal{T}$ should be stationary in $K$, and $K$ itself should have size $\theta$. We continue to describe situations taken from Friedman [2] and Mitchell [6] where this can be done.

Recall that $U \subseteq \theta$ is fat if for every club $B \subseteq \theta, U \cap B$ contains a club of order type $\omega_{1}+1$. For $\alpha<\theta$ of uncountable cofinality, we say that $U$ is locally fat at $\alpha$ if $U \cap \alpha$ contains a countably closed unbounded subset of $\alpha$ or, more precisely, if there is a club $E \subseteq \alpha$ such that $U \cap \alpha \supseteq\{\xi \in E \mid \operatorname{cof}(\xi)=\omega\}$.

Claim 5.3 Let $U$ be a fat subset of $\theta$, which is stationary on points of uncountable cofinality. Then there is $\bar{U} \subseteq U$, still stationary on points of uncountable cofinality, so that for every $\alpha \in \bar{U}$ of uncountable cofinality, $\bar{U}$ is locally fat at $\alpha$. Moreover, $\bar{U}$ can be found such that all its elements have cofinality $\omega$ or $\omega_{1}$.

Proof Let $X=\left\{\alpha \in U \mid \operatorname{cof}(\alpha)=\omega_{1}\right.$ and $U \cap \alpha$ contains a club subset of $\left.\alpha\right\}$. Let $B=\theta-X$. $U \cap B$ cannot contain a club of order type $\omega_{1}+1$, since the top point of such a club would belong to $X$. Since $U$ is fat, it follows that $B$ cannot contain a club, and therefore $X$ is stationary. Set $\bar{U}=X \cup\{\xi \in U \mid \operatorname{cof}(\xi)=\omega\}$.

Recall that $\mathcal{R}$ is a thin stationary subset of $\mathcal{P}_{<\omega_{1}}(\theta)$ if $\mathcal{R}$ is a set of countable subsets of $\theta, \mathcal{R}$ is stationary, and the set $\{\delta<\theta \mid\{x \cap \delta \mid x \in \mathcal{R}\}$ has size $\delta\}$ is unbounded in $\theta$. Equivalently this set is a club in $\theta$ relative to ordinals of uncountable cofinality.

Remark 5.4 If $\delta<\theta \rightarrow \delta^{\omega}<\theta$, then a thin stationary set on $\mathcal{P}_{<\omega_{1}}(\theta)$ exists trivially. Indeed, $\mathcal{P}_{<\omega}(\theta)$ itself is such a set. But thin stationary sets need not always exist (for more on the existence and nonexistence of thin stationary sets see Friedman and Krueger [4]).

Suppose that there exists a thin stationary subset of $\mathcal{P}_{<\omega_{1}}(\theta)$. Let $\mathcal{R}$ be such a set. Let $f: \theta \rightarrow \mathcal{P}_{<\omega_{1}}(\theta)$ be a function so that on a club $Z$ of $\delta<\theta$ of uncountable cofinality, $\{x \cap \delta \mid x \in \mathcal{R}\} \subseteq f^{\prime \prime} \delta$. Such a function can be constructed using the assumption that $\mathcal{R}$ is thin.

Let $U \subseteq \theta$ be stationary on points of uncountable cofinality, and let it be locally fat at all its elements of uncountable cofinality. Let $c$ be a function such that for each $\alpha \in U$ of uncountable cofinality, $c(\alpha) \subseteq \alpha$ is a club witnessing that $U$ is locally fat at $\alpha$.

We show how to add a club subset of $U$, with finite conditions, without collapsing $\omega_{1}, \theta$, or any cardinals above $\theta$. This also shows how to add clubs in fat subsets of $\theta$ which are stationary on points of uncountable cofinality, since any such set can be thinned to $U$ as above using Claim 5.3.

Let $K=L_{\theta}[f, Z, U, c]$ (constructing relative to $Z, U$, and the sets $\{\langle\alpha, \xi\rangle \mid \xi \in$ $f(\alpha)\}$ and $\{\langle\alpha, \xi\rangle \mid \xi \in c(\alpha)\})$. Note that $|K|=\theta$. Let $\mathcal{T}$ consist of transitive $W \in K$ which are elementary in $(K ; f, Z, U)$, with $\sup (W \cap$ Ord $) \in U$ and $\operatorname{cof}(\sup (W \cap$ Ord $))$ uncountable. Let $\delta$ consist of countable $M \in K$ which are elementary in $(K ; f, Z, U)$, with $\sup (M \cap$ Ord $) \in U$, and $M \cap W \in W$ for every $W \in \mathcal{T}$ which belongs to $M$. An argument similar to that in the first paragraph of

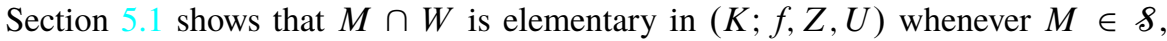


$W \in \mathcal{T}$, and $W \in M$. Moreover, for such $M$ and $W$, $\sup (M \cap W \cap$ Ord $) \in U$ because $U$ is locally fat at $\sup (W \cap$ Ord). (This can be seen as follows. Note that $\sup (W \cap$ Ord $)$ is a point of uncountable cofinality in $U$. A club witnessing local fatness of $U$ at $\sup (W \cap$ Ord) belongs to $M$ by elementarity and inclusion of the function $c$ in the structure $K$. This implies that $\sup (M \cap W \cap \operatorname{Ord})$ belongs to this club, and hence $\sup (M \cap W \cap$ Ord $) \in U$.) Hence $M \cap W \in \delta$. It follows from this, using also the requirement $M \cap W \in W$ in the definition of $\delta$, that $\delta$ and $\mathcal{T}$ are appropriate for $\omega, \omega_{1}$, and $K$.

\section{Claim 5.5 The sets 8 and $\mathcal{T}$ are both stationary.}

Proof The stationarity of $\mathcal{T}$ is immediate from the stationarity of $U$ on points of uncountable cofinality and the fact that $L_{\alpha}[f, Z, U, c] \prec K$ for a club of $\alpha<\theta$. We prove that $\delta$ is stationary. Let $h$ be a function from $K^{<\omega}$ into $K$. We have to prove that there are countable $M$ which belong to $\&$ and are closed under $h$.

Let $W \in \mathcal{T}$ be closed under $h$. Such $W$ exists since $\mathcal{T}$ is stationary. Let $Q \subseteq K$ be countable, elementary in $(K ; f, Z, U)$, and closed under $h$, with $W \in Q$ and $Q \cap$ Ord $\in \mathcal{R}$. Such $Q$ exists since $\mathcal{R}$ is stationary. Set $M=Q \cap W$. Then $M$ is closed under $h$ and elementary in $(K ; f, Z, U)$. The local fatness of $U$ at $\sup (W \cap$ Ord $)$, and the inclusion of a club witnessing this in $K$, imply that $\sup (M \cap$ Ord $)=\sup (Q \cap W \cap$ Ord $) \in U$. Since $Q \cap$ Ord belongs to $\mathcal{R}$ and $\sup (W \cap$ Ord $) \in Z, M \cap$ Ord $=(Q \cap$ Ord $) \cap \sup (W \cap$ Ord $)$ belongs to $f^{\prime \prime} \sup (W \cap$ Ord $) \subseteq W$. Since $M$ can be determined in $K$ from $M \cap$ Ord it follows from this that $M \in K$ and indeed $M \in W$. Moreover, for any $\bar{W} \in \mathcal{T}$ which belongs to $M$, a similar argument shows that $M \cap \bar{W}=Q \cap \bar{W}$ belongs to $\bar{W}$. This establishes that $M \in \mathcal{8}$.

Now by Lemma 5.2, forcing with $\mathbb{P}_{\omega, \delta, \mathcal{T}, K}^{\text {dec }}$ does not collapse $\omega_{1}, \theta$, or any cardinals

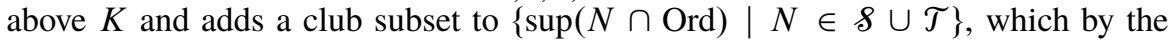
definitions above is contained in $U$.

Remark 5.6 The requirement that $\mathcal{R}$ is thin can be weakened slightly, to require that the sequence of sets $\{x \cap \delta \mid x \in \mathcal{R}$ and $\delta \in x\}$ be approachable on a stationary subset of $U$ of points of uncountable cofinality. Precisely, this means that there is an enumeration $f$ of sets so that for stationarily many $\delta \in U$ of uncountable cofinality, $\{x \cap \delta \mid x \in \mathcal{R}$ and $\delta \in x\} \subseteq f^{\prime \prime} \delta$. (If we removed the part $\delta \in x$, this would be equivalent to thinness.) The proofs above go through essentially unmodified with this condition, restricting $\mathcal{T}$ to $W$ so that $\delta=\sup (W \cap$ Ord $)$ is in the stationary set witnessing approachability. The condition cannot be weakened further since the existence of such $\mathcal{R}$ follows from the existence of stationary $\delta$ and $\mathcal{T}$ which are appropriate for $K$ of size $\theta$. To see this, let $f$ enumerate $K$ in order type $\theta$. Then the set $\{M \cap W \cap$ Ord $\mid M \in \mathcal{S}, W \in \mathcal{T}, M$ and $W$ are elementary with respect to $f$, and $W \in M\}$ is stationary in $\mathcal{P}_{<\omega_{1}}(\theta)$ and approachable on the stationary set $\{\sup (W \cap$ Ord $) \mid W \in \mathcal{T}$ and $W$ is elementary with respect to $f\}$.

5.3 Collapsing without adding branches Let $\kappa$ be a cardinal, and suppose that $\kappa^{<\kappa}=\kappa$. Let $\theta>\kappa^{+}$be regular, with $|H(\theta)|=\theta$, and such that $\delta<\theta \rightarrow \delta^{\kappa}<\theta$.

Set $\mathcal{T}$ to consist of all transitive $W \prec H(\theta)$ so that $|W|<\theta$ and $W$ is $\kappa$-closed. Set $\&$ to consist of all $M \prec H(\theta)$ of size $\kappa$, with $\kappa \in M$, which are closed under 
sequences of length $<\kappa$. Then $\delta$ and $\mathcal{T}$ are appropriate for $\kappa, \kappa^{+}$, and $H(\theta)$. Our assumptions on $\kappa$ and $\theta$ imply that $\delta$ and $\mathcal{T}$ are both stationary.

Let $\mathbb{S}_{\kappa, \theta}$ be the poset $\mathbb{P}_{\kappa, \delta, \mathcal{T}, H(\theta)}$. By arguments similar to the collapsing arguments in Section 5.1, forcing with $\mathbb{S}_{\kappa, \theta}$ collapses all cardinals between $\kappa^{+}$and $\theta$ to $\kappa^{+}$. By Claim 2.8, $\mathbb{S}_{\kappa, \theta}$ is $<\kappa$-closed. It follows that forcing with $\mathbb{S}_{\kappa, \theta}$ does not collapse $\kappa$ or any smaller cardinals and indeed does not add sequences of ordinals of length $<\kappa$. By Claim $4.1, \mathbb{S}_{\kappa, \theta}$ is strongly proper for $\delta \cup \mathcal{T}$. Since $\delta$ and $\mathcal{T}$ are both stationary, it follows using Remarks 3.2 and 3.1 and Claim 3.3 that forcing with $\mathbb{S}_{\kappa, \theta}$ does not collapse $\kappa^{+}$and $\theta$. Since $\mathbb{S}_{\kappa, \theta}$ has size $|H(\theta)|=\theta$, it does not collapse cardinals or change cofinalities above $\theta$.

Altogether then, $\mathbb{S}_{\kappa, \theta}$ has the same collapsing effects as $\operatorname{Col}\left(\kappa^{+},<\theta\right)$.

The collapse poset $\operatorname{Col}\left(\kappa^{+},<\theta\right)$ can be split into forcing first with an initial segment $\operatorname{Col}\left(\kappa^{+}, \gamma\right)$ and then with a tail end $\operatorname{Col}\left(\kappa^{+},[\gamma, \theta)\right)$. A similar splitting is possible with $\mathbb{S}_{\kappa, \theta}$. For any $W \in \mathcal{T}$, forcing with $\mathbb{S}_{\kappa, \theta}$ below the condition $\{W\}$ is, by strong properness, the same as forcing first with $\mathbb{S}_{\kappa, \theta} \cap W$ and then with the factor poset $\mathbb{S}_{\kappa, \theta} /\left(\mathbb{S}_{\kappa, \theta} \cap W\right)$. (Recall that the factor poset consists of all conditions $s \in \mathbb{S}_{\kappa, \theta}$ so that $W \in s$ and $\operatorname{res}_{W}(s)$ belongs to the generic added by $\mathbb{S}_{\kappa, \theta} \cap W$.)

However, in contrast with $\operatorname{Col}\left(\kappa^{+},<\theta\right), \mathbb{S}_{\kappa, \theta}$ does not add branches of length $\kappa^{+}$ or greater through trees in $V$. More precisely, if $T$ is a tree in $V, G$ is generic for $\mathbb{S}_{\kappa, \theta}$ over $V$, and $f$ is a branch of $T$ that belongs to $V[G]$ and has length $\geq \kappa^{+}$, then $f \in V$. This follows from strong properness for $\delta$ and the stationarity of $\delta$, by Lemma 3.7. Furthermore, the factor posets $\mathbb{S}_{\kappa, \theta} /\left(\mathbb{S}_{\kappa, \theta} \cap W\right)$ too do not add branches of length $\geq \kappa^{+}$to trees in the extension of $V$ by $\mathbb{S}_{\kappa, \theta} \cap W$. This again follows by Lemma 3.7 , this time using strong properness of the factor poset, given by Claims 4.3 and 4.4 .

(Note that the ordinary collapse poset, $\operatorname{Col}\left(\kappa^{+},<\theta\right)$, does add new branches of length $\kappa^{+}$through trees in $V$. Indeed, every segment $\operatorname{Col}\left(\kappa^{+}, \gamma\right)$ of the poset adds such a branch, for example, through the tree of functions into $\gamma$ with domain $<\kappa^{+}$, ordered by extension.)

This property of $\mathbb{S}_{\kappa, \theta}$ makes it a useful substitute for the ordinary collapse in arguments that deal with the tree property.

One example involves models of the tree property at successors of singular cardinals together with failure of the singular cardinals hypothesis. Neeman [8] produced such models for large singular cardinals. Sinapova [10] then produced such models for $\aleph_{\omega^{2}}$. Passing from Neeman's construction to Sinapova's requires several collapses that ultimately will turn the large cardinal at the starting point to $\aleph_{\omega^{2}}$. The initial impediment for combining such collapses with Neeman's construction is in adapting [8, Lemma 3.2], which absorbs a branch through a tree from a generic extension of a model to the model itself. Later impediments are of a similar nature.

Sinapova overcame these impediments in [10] using a very clever argument on narrow systems and systems of branches through them. If one were to replace the ordinary collapses used in the construction, by the model sequence collapses defined above, then less clever arguments would suffice. (The parallel of [8, Lemma 3.2] for settings with the collapse is a consequence of the "no new branches" property for factors of the model sequence collapses. Similar arguments, and other arguments using closure of the model sequence collapses, can handle later issues in the adaptation.) 
5.4 A particular model of PFA (after Friedman [3]) Veličković [12, Theorem 3.13] proves that under the semiproper forcing axiom (SPFA), every inner model which is correct about $\omega_{2}$ must contain all the reals. It is natural to ask if the same result holds with PFA. Veličković and Caicedo [1] show that a variant holds under PFA; namely, that if the inner model itself also satisfies PFA (and is correct about $\omega_{2}$ ), then it must contain all reals. Indeed they prove this also for the bounded proper forcing axiom, BPFA. However the original result fails under PFA. Friedman [3] obtains a model of PFA with an inner model that is correct about $\omega_{2}$ but does not contain all reals (and similarly of BPFA, from smaller large cardinal assumptions). His construction uses the side conditions of Friedman [2]. We briefly sketch an adaptation of the construction to use the two-type model sequences.

Let $\theta$ be a supercompact cardinal. Let $K=H(\theta)$. Let 8 consist of all countable elementary substructures of $K$. Let $Z=\{\alpha<\theta \mid H(\alpha) \prec H(\theta)$ and $H(\alpha)$ is countably closed $\}$. Here $\alpha$ ranges over cardinals, and similarly in all contexts below when we talk about $H(\alpha)$. Let $\mathcal{T}=\{H(\alpha) \mid \alpha \in Z\} .8$ and $\mathcal{T}$ are appropriate for $\omega, \omega_{1}$, and $K$. Let $\mathbb{P}=\mathbb{P}_{\omega, \delta, \mathcal{T}, K}$. Let $G$ be generic for $\mathbb{P}$ over $V$. As in Sections 5.1 and 5.2, $\theta$ is turned to $\omega_{2}$ in the extension $V[G]$, and apart from this no cardinals are collapsed.

We intend to work with the set $\{\alpha \mid H(\alpha) \in \bigcup G\}$. As in previous subsections, the set is a club in $\theta$ relative to $Z$, and this is all we need for the argument on PFA. But in fact, in this case, the set is outright equal to $Z$. In other words, for every $\alpha \in Z, H(\alpha) \in G$. This is immediate by a density argument using the following claim.

Claim 5.7 Suppose that $\mathcal{T}$ consists exactly of nodes $H(\alpha) \prec H(\theta)$ so that $\alpha<\theta$ and $H(\alpha)$ is countably closed. Suppose also that all $M \in \S$ are elementary in $H(\theta)$.

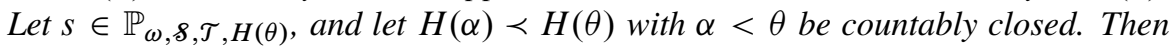
there is $r \leq s$ with $H(\alpha) \in r$.

Moreover, one can arrange in addition that all new nodes in $r$, meaning nodes in $r-s$, are either of transitive type or of the form $N \cap W$, where $N \in s$ and $W \in \mathcal{T}$.

Proof Fix $s$ and $\alpha$. If $s \subseteq H(\alpha)$, then $s \cup\{H(\alpha)\}$ is a condition. Suppose then that $s \nsubseteq H(\alpha)$, and let $M$ be the first node of $s$ outside $H(\alpha)$. If $M=H(\alpha)$, take $r=s$. If $\alpha \in M$, then $\operatorname{res}_{M}(s) \cup\{H(\alpha)\}$ is a condition that belongs to $M$ and extends $\operatorname{res}_{M}(s)$. By Corollary 2.31 it is directly compatible with $s$, giving an extension $r$ of $s$ which includes $H(\alpha)$. Since $r$ is the closure of $s \cup\{H(\alpha)\}$ under intersections, the new nodes in $r$ are $H(\alpha)$ itself and intersections of small nodes of $s$ with $H(\alpha)$.

Suppose finally that $M$ is above $H(\alpha)$ and $\alpha \notin M$. In particular, $M$ is of small type. Let $\alpha^{*}$ be the first ordinal in $M$ above $\alpha$. Such an ordinal exists since $M \nsubseteq H(\alpha)$. The minimality of $\alpha^{*}$ implies that $M \cap H\left(\alpha^{*}\right)=M \cap H(\alpha)$. From this, the elementarity of $M$, and the elementarity of $H(\alpha)$, it follows that $H\left(\alpha^{*}\right) \prec H(\theta)$. (Suppose not. Then there are $a_{1}, \ldots, a_{n} \in H\left(\alpha^{*}\right)$ and a formula $\varphi$ so that in $H(\theta)$ there exists some $y$ so that $H(\theta) \models \varphi\left(a_{1}, \ldots, a_{n}, y\right)$, but there is no such $y$ in $H\left(\alpha^{*}\right)$. By elementarity of $M, a_{1}, \ldots, a_{n}$ can be found in $M$. They then belong to $H(\alpha)$. By elementarity of $H(\alpha), y$ can be found in $H(\alpha)$ and hence also in $H\left(\alpha^{*}\right)$.) It also follows that $\alpha^{*}$ has uncountable cofinality. So $H\left(\alpha^{*}\right) \in \mathcal{T}$. The argument of the previous paragraph shows that there is an extension $s^{*}$ of $s$ with $H\left(\alpha^{*}\right) \in s^{*}$. The first node of $s^{*}$ above $\alpha$ has smaller von Neumann rank than the first node of $s$ above $\alpha$, and by induction it follows that there is an extension $r^{*}$ of $s^{*}$, hence also 
of $s$, that contains $H(\alpha)$. In each of these two steps, the new nodes in the extension are either of transitive type or of the form $N \cap W$, where $N \in s$ and $W \in \mathcal{T}$, using the argument of the previous paragraph for the first step and induction for the second.

Remark 5.8 Let $\left\langle H(\theta) ; A_{1}, \ldots, A_{k}\right\rangle$ be an expansion of $H(\theta)$ by finitely many predicates. Then in Claim 5.7 and its proof, elementarity in $H(\theta)$ can be replaced by elementarity in $\left\langle H(\theta) ; A_{1}, \ldots, A_{k}\right\rangle$ throughout.

We now briefly sketch Friedman's argument to force over $V[G]$ to obtain PFA, without collapsing any cardinals. The forcing will add reals, and so in the resulting model of PFA, $V[G]$ will be a submodel that is correct about $\omega_{2}$ and does not include all reals.

We work throughout over the model $V[G]$. Our intention is to build a Laver iteration $\mathbb{R}$ of length $\theta=\omega_{2}^{V[G]}$ over $V[G]$, which forces PFA and does not collapse any cardinals. To reach PFA in the extension, call it $V[G][I]$, we have to allow, at stages $\alpha<\theta$ of the iteration, posets which are proper in $V[G \cap H(\alpha)][I \uparrow \alpha]$. One of the key points in the argument is that properness transfers from $V[G \cap H(\alpha)][I \uparrow \alpha]$ to $V[G][I \uparrow \alpha]$, allowing us to argue that the iteration is proper over $V[G]$.

Let $F \in V$ be a Laver function for the supercompact cardinal $\theta$. Let $\theta^{*}>\theta$, and let $8^{*}$ be the set of countable $M \prec H\left(\theta^{*}\right)$ with $\theta, F \in M$. For each $\alpha \in Z \cup\{\theta\}$, let $\delta_{\alpha}^{*}=\left\{M[G \cap H(\alpha)] \mid M \in \delta^{*}, \alpha \in M\right.$, and $\left.M \cap H(\alpha) \in G\right\}$. Let $\mathbb{Q}_{\alpha}$ be the factor poset for adding $G$ over $V[G \cap H(\alpha)]$. By Claim 4.4 and the comment following the claim, $\mathbb{Q}_{\alpha}$ is strongly proper for $8_{\alpha}^{*}$.

A diagonal countable support iteration of length $\theta$ over $V[G]$, leading to a final poset $\mathbb{R}$, say, is the variant of a countable support iteration defined by placing the following additional restriction on the sequences $r$ that are allowed as conditions in $\mathbb{R}$ and in its initial segments $\mathbb{R} \uparrow \alpha$ : for every $\beta$ that belongs to $Z, r \uparrow \beta$ must belong to $V[G \cap H(\beta)]$. (In an ordinary iteration over $V[G]$ one would have only $r \uparrow \beta \in V[G]$.) The notion is due to Friedman [3].

Let $\bar{Z} \subseteq Z$ be the set of inaccessible $\alpha$ so that $H(\alpha)$ is closed under $F$. Working in $V[G]$, let $\mathbb{R}$ be the diagonal countable support length $\theta$ iteration of the posets given by $\mathbb{R} \uparrow \alpha$-names $F(\alpha)[G \cap H(\alpha)]$ for $\alpha \in \bar{Z}$ so that, in $\mathbb{R} \uparrow \alpha$ over $V[G \cap H(\alpha)]$, it is forced that $F(\alpha)[G \cap H(\alpha)]$ is proper with respect to (the $\mathbb{R} \uparrow \alpha$ extensions of models in) $\wp_{\alpha}^{*}$. ( $\mathbb{R}$ is similar to the poset $Q$ in Friedman [3].)

The use of diagonal iteration in the definition of $\mathbb{R}$, together with the fact that the individual posets being iterated all have size $<\theta$, implies that $\mathbb{R}$ is $\theta$-c.c. over $V[G]$. The proof of this involves a standard $\Delta$-system argument; we note only that the use of a diagonal iteration is essential for the argument as it limits the number of conditions with any fixed support to less than $\theta$. Since $\mathbb{R}$ is $\theta$-c.c. over $V[G]$, it does not collapse any cardinals $\geq \omega_{2}^{V[G]}=\theta$.

Recall that $8_{\alpha}^{*}$ consists of structures of the form $M[G \cap H(\alpha)]$. By definition $\mathbb{R}$ only uses $F(\alpha)[G \cap H(\alpha)]$ if it is forced to be proper for these structures. The next claim shows that $F(\alpha)[G \cap H(\alpha)]$ is then forced to be proper also for the extended structures $M[G]$.

Claim 5.9 Let $\alpha$ be as above, and let $M \prec H\left(\theta^{*}\right)$ be countable with $\alpha, F \in M$. Then it is forced, in $\mathbb{R} \uparrow \alpha$ over $V[G]$, that $F(\alpha)[G \cap H(\alpha)]$ is proper for $M[G]$. 
Proof This follows by condition (3) in Claim 3.8, using the fact that the factor poset $\mathbb{Q}_{\alpha}$, which leads from $V[G \cap H(\alpha)]$ to $V[G]$, is strongly proper for $M[G \cap H(\alpha)]$ and remains so in the extension of $V[G \cap H(\alpha)]$ by $\mathbb{R} \uparrow \alpha$. Claim 3.8 is used with the model $M[G \cap H(\alpha)][I]$ in $V[G \cap H(\alpha)][I]$, where $I$ is generic for $\mathbb{R} \uparrow \alpha$ over $V[G \cap H(\alpha)]$. (In passing from $M[G \cap H(\alpha)]$ to $M[G \cap H(\alpha)][I]$ we make implicit use of the properness of $\mathbb{R} \uparrow \alpha$ for $M[G \cap H(\alpha)]$, which is proved below. Formally the two proofs are by simultaneous induction.)

The poset $\mathbb{R}$ is, by the last claim, a countable support diagonal iteration of proper posets in $V[G]$, with properness restricted to elementary substructures in $\delta_{\theta}^{*}$. Were it not for the use of a diagonal iteration, preservation of properness under countable support iterations would directly imply that $\mathbb{R}$ is itself proper for these substructures. With the use of a diagonal iteration, at each limit stage $\gamma$, the inductive step of the preservation argument only yields that $\mathbb{R} \uparrow \gamma$ is proper for structures $M\left[G \cap H\left(\alpha^{*}\right)\right]$ in $V\left[G \cap H\left(\alpha^{*}\right)\right]$, where $\alpha^{*}$ is the first element of $Z$ that is at least $\gamma$. Fortunately, by condition (3) of Claim 3.8 this implies properness of $\mathbb{R} \uparrow \gamma$ for structures $M[G]$ in $V[G]$, allowing the inductive preservation argument to proceed and showing ultimately that $\mathbb{R}$ is proper in $V[G]$ for structures in $\wp_{\theta}^{*}$. In particular, then $\mathbb{R}$ does not collapse $\omega_{1}$ over $V[G]$.

Let $I$ be generic for $\mathbb{R}$ over $V[G]$. We have seen above that $V[G][I]$ and $V[G]$ have the same cardinals. It is clear that in $V[G][I]$ there are more reals. Standard arguments, directly from the definition of $\mathbb{R}$, using the supercompactness of $\theta$ and the fact that $F$ is a Laver function, show that PFA holds in $V[G][I]$.

\section{The Consistency of PFA Using Finite Supports}

Our main application of the sequence models poset is a new proof of the consistency of the proper forcing axiom, which does not use preservation of properness under countable support iterations. Instead, we will work with a finite support iteration and use side conditions from the sequence poset to enforce properness.

Let $\theta$ be a supercompact cardinal. Let $F: \theta \rightarrow H(\theta)$ be a Laver function. Set $K=H(\theta)$. Let $Z$ be the set of $\alpha<\theta$ so that $(H(\alpha) ; F \uparrow \alpha)$ is elementary in $(H(\theta) ; F)$. For each $\alpha \in Z$, let $f(\alpha)$ be the least cardinal so that $F(\alpha) \in H(f(\alpha))$. Note that $f(\alpha)$ is smaller than the next element of $Z$ above $\alpha$. Set $\mathcal{T}$ to be the set of models $W=H(\alpha)$ for $\alpha \in Z$ so that $H(\alpha)$ is countably closed (equivalently, $\alpha$ has uncountable cofinality). Set $\delta$ to be the set of countable models which are elementary in $(H(\theta) ; F) . \delta$ and $\mathcal{T}$ are then stationary and appropriate for $\omega, \omega_{1}$, and $K=H(\theta)$.

We describe a poset $\mathbb{A}$ that forces PFA. Conditions in the poset have two components. One corresponds to a finite support iteration of proper posets given by the Laver function $F$. This is similar to the standard consistency proof for PFA, except that finite supports are used instead of countable. The other component consists simply of conditions in the model sequence poset $\mathbb{P}_{\omega, \delta, \mathcal{T}, H(\theta)}$. We will connect the two components by restricting conditions in the first to master conditions for models in the second.

Definition 6.1 Conditions in the poset $\mathbb{A}$ are pairs $\langle s, p\rangle$ so that

(1) $s$ is a condition in $\mathbb{P}_{\omega, \delta, \mathcal{T}, H(\theta)}$; in other words, it is a finite, $\in$-increasing sequence of models from $\delta \cup \mathcal{T}$, closed under intersections; 
(2) $p$ is a partial function on $\theta$, with domain contained in the (finite) set $\left\{\alpha<\theta \mid H(\alpha) \in s\right.$ and $\vdash_{\mathbb{A} \cap H(\alpha)}$ " $F(\alpha)$ is a proper poset" $\}$;

(3) for all $\alpha \in \operatorname{dom}(p), p(\alpha) \in H(f(\alpha))$;

(4) for all $\alpha \in \operatorname{dom}(p), \vdash_{\mathbb{A} \cap H(\alpha)} p(\alpha) \in F(\alpha)$;

(5) for all $\alpha \in \operatorname{dom}(p)$ and each small node $M \in s$ so that $\alpha \in M$, $\langle s \cap H(\alpha), p \uparrow \alpha\rangle \vdash_{\mathbb{A} \cap H(\alpha)}$ " $p(\alpha)$ is a master condition for $M$."

The ordering on $\mathbb{A}$ is the following: $\left\langle s^{*}, p^{*}\right\rangle \leq\langle s, p\rangle$ iff $s^{*} \leq s$ and for every $\alpha \in \operatorname{dom}(p),\left\langle s^{*} \cap H(\alpha), p^{*} \uparrow \alpha\right\rangle \Vdash_{\mathbb{A} \cap H(\alpha)} p^{*}(\alpha) \leq_{F(\alpha)} p(\alpha)$.

To avoid confusion in the arguments below, we mostly use the same pairings of letters for conditions: we will use pairs $\langle s, p\rangle,\langle t, q\rangle,\langle u, h\rangle$, and $\langle a, h\rangle$ and will mostly avoid other pairings such as $\langle s, q\rangle$ or $\langle t, p\rangle$.

Definition 6.1 is an induction on $\alpha \in Z \cup\{\theta\}$, as knowledge of $\mathbb{A} \cap H(\alpha)$ is needed to evaluate the conditions on $p(\alpha)$. Conditions (2)-(4) are the standard conditions in an iteration of proper posets given by the Laver function $F$. Condition (5)

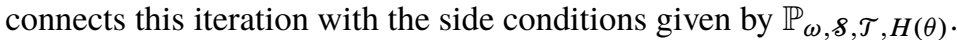

Remark 6.2 If $\alpha \in Z$, then $\mathbb{A} \cap H(\alpha)$ is definable in $(H(\theta) ; F)$ from $\alpha$. This is because $F \uparrow \alpha$ is definable, and so are $\delta \cap H(\alpha)$ and $\mathcal{T} \cap H(\alpha)$. (The parts of $\delta$ and $\mathcal{T}$ below $\alpha$ can be defined using elementarity in $(H(\alpha) ; F \uparrow \alpha)$ instead of elementarity in $(H(\theta) ; F)$, and this can be done inside $(H(\theta) ; F)$ with $\alpha$ as parameter.) In particular, it follows that $\mathbb{A} \cap H(\alpha) \in M$ for every $M \in \mathcal{S}$ with $\alpha \in M$.

Remark 6.3 Condition (5) involves some abuse of notation, since $M$ is not an elementary substructure of the extension of $H(\theta)$ by $\mathbb{A} \cap H(\alpha)$. What we mean precisely is that $\langle s \cap H(\alpha), p \uparrow \alpha\rangle \vdash_{\mathbb{A} \cap H(\alpha)}$ " $p(\alpha)$ is a master condition for $M\left[\dot{G}_{\alpha}\right]$ " where $\dot{G}_{\alpha}$ names the $\mathbb{A} \cap H(\alpha)$ generic.

Remark 6.4 Condition (5) holds for $\alpha$ and $M$ iff it holds for $\alpha$ and $M \cap H(\gamma)$, whenever $\gamma \in Z \cup\{\theta\}$ is larger than $\alpha$. The reason is that $F(\alpha) \in H(f(\alpha)) \subseteq H(\gamma)$, and so being a master condition for $M$ in the interpretation of $F(\alpha)$ is equivalent to being a master condition for $M \cap H(\gamma)$.

Claim 6.5 Condition (5) in Definition 6.1 is equivalent to the same condition with the restriction to " $M \in S$ so that $\alpha \in M$ " replaced by the restriction to " $M \in s$ which occur above $H(\alpha)$ in $s$ and so that there are no transitive nodes in $s$ between $H(\alpha)$ and $M . "$

Proof It is clear that the original condition implies the version in the claim, since by Claim 2.10, $\alpha \in M$ for every $M$ which occurs above $H(\alpha)$ in $s$ with no transitive nodes between $H(\alpha)$ and $M$. For the converse, let $W^{*}$ be the first transitive node of $s$ above $H(\alpha)$ if there is one, and $H(\theta)$ otherwise. By Remark 6.4, being a master condition for $M$ in $F(\alpha)$ is equivalent to being a master condition for $M \cap W^{*}$. From this, the closure of $s$ under intersection with $W^{*}$, and the fact that if $\alpha \in M$, then $\alpha \in M \cap W^{*}$ and hence $M \cap W^{*}$ occurs above $H(\alpha)$, it follows that being a master condition for all $M \in s$ with $\alpha \in M$ is a consequence of being a master condition for all $M \in s$ between $H(\alpha)$ and $W^{*}$.

For $\beta \in Z \cup\{\theta\}$, let $\mathbb{A}_{\beta}$ denote the poset given by Definition 6.1 with the added restriction that $\operatorname{dom}(p) \subseteq \beta$. For $\beta \in Z$ this poset is related to $\mathbb{A} \cap H(\beta)$, but the two are not the same, since the latter restricts the side conditions to belong to $H(\beta)$, while the former does not. $\mathbb{A}_{\theta}$ is equal to $\mathbb{A}$. 
Claim 6.6 Let $\alpha<\beta$ belong to $Z \cup\{\theta\}$. Let $\langle s, p\rangle \in \mathbb{A}_{\beta}$ with $W=H(\alpha) \in s$. Let $\langle t, q\rangle \in \mathbb{A} \cap H(\alpha)$ extend $\langle s \cap H(\alpha), p \uparrow \alpha\rangle$. Then $\langle s, p\rangle$ and $\langle t, q\rangle$ are compatible in $\mathbb{A}_{\beta}$. Moreover, this is witnessed by the condition $\langle u, h\rangle$ with $u=t \cup s$, $h \uparrow \alpha=q$, and $h \uparrow[\alpha, \beta)=p \uparrow[\alpha, \beta)$.

Proof $s \cap H(\alpha)$ is equal to $\operatorname{res}_{W}(s)$, so by Corollary 2.31, $t$ and $s$ are directly

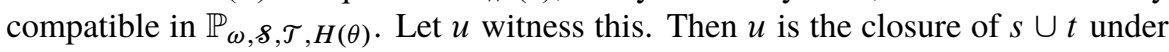
intersections, and since $t$ extends an initial segment of $s, u$ is simply equal to $s \cup t$.

Let $h=q \cup p \uparrow[\alpha, \beta)$. It is easy, with some uses of Claim 6.5, to check that $\langle u, h\rangle$ is a condition in $\mathbb{A}_{\beta}$ and that it extends both $\langle t, q\rangle$ and $\langle s, p\rangle$.

Lemma 6.7 Let $\beta$ belong to $Z \cup\{\theta\}$.

(1) Let $\langle s, p\rangle \in \mathbb{A}_{\beta}$, and let $W=H(\alpha)$ be a transitive node in $s$. Then $\langle s, p\rangle$ is a strong master condition for $W$ in $\mathbb{A}_{\beta}$.

(2) Let $\langle s, p\rangle \in \mathbb{A}_{\beta}$, let $W \in \mathcal{T}$, and suppose $\langle s, p\rangle \in W$. Then $\langle s \cup\{W\}, p\rangle$ is a condition in $\mathbb{A}_{\beta}$. (It trivially extends $\langle s, p\rangle$.)

(3) $\mathbb{A}_{\beta}$ is strongly proper for $\mathcal{T}$.

Proof Condition (2) is immediate from the definitions, using Corollary 2.32,

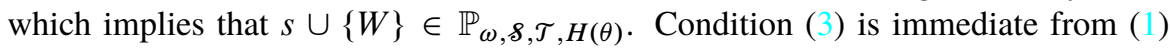
and (2). Condition (1) in the case when $\alpha<\beta$ follows from Claim 6.6. Let $D$ be dense in $\mathbb{A}_{\beta} \cap H(\alpha)$. It is enough to prove that every $\langle s, p\rangle \in \mathbb{A}_{\beta}$ with $H(\alpha) \in s$ is compatible with a condition in $D$. Fix $\langle s, p\rangle$. By density of $D$ in $\mathbb{A}_{\beta} \cap H(\alpha)$, there is $\langle t, q\rangle \in D$ which extends $\langle s \cap H(\alpha), p \uparrow \alpha\rangle$. By Claim 6.6, $\langle s, p\rangle$ and $\langle t, q\rangle$ are compatible, and the condition $\langle u, h\rangle$ given by the claim to witness this belongs to $\mathbb{A}_{\beta}$. In the case $\alpha \geq \beta$, condition (1) is proved similarly with a direct use of Corollary 2.31 instead of Claim 6.6.

Claim 6.8 Let $\mathbb{Q}$ be proper. Let $\kappa$ be large enough that $\mathbb{Q} \in H(\delta)$ for some $\delta<\kappa$. Let $M_{0} \in M_{1} \in \cdots \in M_{l-1}$ be countable elementary substructures of $H(\kappa)$ with $\mathbb{Q} \in M_{i}$ for all $i$. Suppose that $k<l$ and $q \in M_{k}$ is a master condition for $M_{0}, \ldots, M_{k-1}$. Then there is $q^{*} \leq q$ which is a master condition for $M_{0}, \ldots, M_{l-1}$.

Proof By standard arguments using the fact that $\kappa$ is larger than the least $\delta$ with $\mathbb{Q} \in H(\delta)$, for every countable $M \prec H(\kappa)$, and every $r \in M$, there is $r^{*} \leq r$ which is a master condition for $M$. The claim follows by successive applications of this fact, setting $r_{k}=q \in M_{k}$, obtaining for each $i \geq k$ a master condition $r_{i+1} \leq r_{i}$ for $M_{i}$ in $M_{i+1}$, and taking $q^{*}=r_{l} ; r_{i+1}$ can be found in $M_{i+1}$ by elementarity.

Claim 6.9 Let $\langle s, p\rangle \in \mathbb{A}$. Suppose $H(\alpha) \in s$ and $\alpha \notin \operatorname{dom}(p)$. Let $M$ be a small node of $s$, and let $\langle t, q\rangle \in \mathbb{A} \cap M$. Suppose that $\alpha \in \operatorname{dom}(q)$ and $\langle s, p\rangle \leq\langle t, q \uparrow \theta-\{\alpha\}\rangle$. Suppose further that $\operatorname{res}_{M}(s)-H(\alpha) \subseteq t$. Then there is $p^{\prime}$ extending $p$ with $\operatorname{dom}\left(p^{\prime}\right)=\operatorname{dom}(p) \cup\{\alpha\}$ and such that $\left\langle s, p^{\prime}\right\rangle$ is a condition in $\mathbb{A}$ extending $\langle t, q\rangle$.

Proof Let $W$ be the first transitive node of $s$ above $H(\alpha)$ if there is one, and $H(\theta)$ otherwise. Let $N_{0} \in \cdots \in N_{l-1}$ list the small nodes of $s$ above $H(\alpha)$ and below $W$. Since $q \in M$, $\operatorname{dom}(q)$ is finite, and $\alpha \in \operatorname{dom}(q)$, we have $\alpha \in M$. This implies that $M \cap W$ appears on the list $N_{0}, \ldots, N_{l-1}$. Fix $k$ so that $M \cap W=N_{k}$. Since $\langle t, q\rangle \in \mathbb{A}$ and $\alpha \in \operatorname{dom}(q), \Vdash_{\mathbb{A} \cap H(\alpha)}$ " $F(\alpha)$ is a proper poset and $q(\alpha) \in F(\alpha)$." Moreover, for every small node $N \in t$ with $\alpha \in N$, it is forced by $\langle t \cap H(\alpha), q \uparrow \alpha\rangle$ 
and hence also by $\langle s \cap H(\alpha), p \uparrow \alpha\rangle$, that $q(\alpha)$ is a master condition for $N$. In particular, since $t \supseteq \operatorname{res}_{M}(s)-H(\alpha) \supseteq\left\{N_{0}, \ldots, N_{k-1}\right\}$, this holds for $N_{0}, \ldots, N_{k-1}$.

By Claim 6.8 there is a name $\dot{q}^{*}$ which is forced by $\langle s \cap H(\alpha), p \uparrow \alpha\rangle$ to be a master condition for all models $N_{0}, \ldots, N_{l-1}$ and to extend $q(\alpha) ; \dot{q}^{*}$ can be picked in $H(f(\alpha))$.

Set $p^{\prime}=p \cup\left\{\alpha \mapsto \dot{q}^{*}\right\}$. It is easy using Claim 6.5 to check that $\left\langle s, p^{\prime}\right\rangle$ is a condition. It extends $\langle t, q\rangle$ by the claim assumption that $\langle s, p\rangle \leq\langle t, q \uparrow \theta-\{\alpha\}\rangle$.

Claim 6.10 Let $\langle s, p\rangle,\langle t, q\rangle \in \mathbb{A}$. Let $M$ be a small node of $s$, and suppose that $\langle t, q\rangle \in M$. Suppose that for some $\delta<\theta,\langle s, p\rangle$ extends $\langle t, q \uparrow \delta\rangle$ and $\operatorname{dom}(q)-\delta$ is disjoint from $\operatorname{dom}(p)$. Suppose further that $\operatorname{res}_{M}(s)-H(\delta) \subseteq t$. Then there is $p^{\prime}$ extending $p$ so that $\operatorname{dom}\left(p^{\prime}\right)=\operatorname{dom}(p) \cup(\operatorname{dom}(q)-\delta)$ and so that $\left\langle s, p^{\prime}\right\rangle$ is a condition in $\mathbb{A}$ extending $\langle t, q\rangle$.

Proof Immediate by successive applications of Claim 6.9, going over all $\alpha \geq \delta$ in $\operatorname{dom}(q)$ in increasing order.

Corollary 6.11 Let $M$ be a small node, and let $\langle t, q\rangle \in \mathbb{A} \cap M$. Then there is $\langle s, p\rangle \leq\langle t, q\rangle$ with $M \in s$.

Proof By Lemma 2.21, $\{M\}$ and $t$ are directly compatible. Let $s$ witness this. Note that $\operatorname{res}_{M}(s)=t$ by Corollary 2.31. Now apply Claim 6.10 with $\delta=0$ to $\langle s, \varnothing\rangle$ and $\langle t, q\rangle$.

Lemma 6.12 Let $\beta \in Z \cup\{\theta\}$. Let $\langle s, p\rangle$ be a condition in $\mathbb{A}_{\beta}$. Let $\theta^{*}>\theta$, and let $M^{*} \prec H\left(\theta^{*}\right)$ be countable with $\theta, F, \beta \in M^{*}$. Let $M=M^{*} \cap H(\theta)$, and suppose that $M \in s$. Then we have the following.

(1) For every $D \in M^{*}$ which is dense in $\mathbb{A}_{\beta}$, there is $\langle t, q\rangle \in D \cap M^{*}$ which is compatible with $\langle s, p\rangle$. Moreover, there is $\left\langle s^{*}, p^{*}\right\rangle \in \mathbb{A}_{\beta}$ extending both $\langle s, p\rangle$ and $\langle t, q\rangle$, so that $\operatorname{res}_{M}\left(s^{*}\right)-H(\beta) \subseteq t$, and all small nodes of $s^{*}$ above $\beta$ and outside $M$ are either nodes of sor of the form $N^{\prime} \cap W$, where $N^{\prime}$ is a small node of $s$ and $W \in \mathcal{T}$;

(2) $\langle s, p\rangle$ is a master condition for $M^{*}$ in $\mathbb{A}_{\beta}$.

Proof Condition (2) for $\langle s, p\rangle$ is immediate from condition (1) for all extensions of $\langle s, p\rangle$. We prove condition (1) by induction on $\beta$. If $\beta$ is the first element of $Z$, then $\mathbb{A}_{\beta}$ is isomorphic to $\mathbb{P}_{\omega, \delta, \mathcal{T}, H(\theta)}$ and the condition holds by Corollary 2.31. We handle the limit and successor cases below.

Suppose first that $\beta$ is a limit point of $Z \cup\{\theta\}$. Let $\bar{\beta}=\sup \left(\beta \cap M^{*}\right)$. This may be $\beta$ itself if $\operatorname{cof}(\beta)=\omega$. Let $\delta<\bar{\beta}$ in $Z \cap M^{*}$ be large enough that $\operatorname{dom}(p) \cap \bar{\beta} \subseteq \delta$. Such $\delta$ exists since $\operatorname{dom}(p)$ is finite, while $\bar{\beta}$ is a limit point of $Z \cap M$.

Let $E$ be the set of conditions $\langle t, \bar{q}\rangle \in \mathbb{A}_{\delta}$ which extend to conditions $\langle t, q\rangle \in D$ with $q \uparrow \delta=\bar{q} . \quad E$ is dense in $\mathbb{A}_{\delta}$ and belongs to $M^{*}$. By induction there is $\langle t, \bar{q}\rangle \in E \cap M^{*}$ which is compatible with $\langle s, p \uparrow \delta\rangle$. Moreover, there is $\left\langle s^{*}, p_{1}\right\rangle \in \mathbb{A}_{\delta}$ which extends both $\langle t, \bar{q}\rangle$ and $\langle s, p \uparrow \delta\rangle=\langle s, p \uparrow \bar{\beta}\rangle$, with $\operatorname{res}_{M}\left(s^{*}\right)-H(\delta) \subseteq t$, and so that all small nodes of $s^{*}$ above $\delta$ and outside $M$ are either nodes of $s$ or of the form $N^{\prime} \cap W$, where $N^{\prime}$ is a small node of $s$ and $W \in \mathcal{T}$. 
Let $\langle t, q\rangle \in D$ witness that $\langle t, \bar{q}\rangle \in E$. Using elementarity of $M^{*}$, pick $q$ inside $M^{*}$. By Claim 6.10, there is $p_{2}$ extending $p_{1}$ so that $\operatorname{dom}\left(p_{2}\right)=\operatorname{dom}\left(p_{1}\right) \cup$ $(\operatorname{dom}(q)-\delta)$ and so that $\left\langle s^{*}, p_{2}\right\rangle$ extends $\langle t, q\rangle$.

Set $p^{*}=p_{2} \cup p \uparrow[\bar{\beta}, \beta)$. It is enough now to prove that $\left\langle s^{*}, p^{*}\right\rangle$ is a condition in $\mathbb{A}_{\beta}$. (By construction it extends both $\langle t, q\rangle$ and $\langle s, p\rangle$.) Fix $\alpha \in \operatorname{dom}(p) \uparrow[\bar{\beta}, \beta)$, and fix a small node $N \in s^{*}$ with $\alpha \in N$. We must check that $p^{*}(\alpha)=p(\alpha)$ is forced by $\left\langle s^{*} \cap H(\alpha), p^{*} \uparrow \alpha\right\rangle$ to be a master condition for $N$. It is enough to check that this is forced by $\langle s \cap H(\alpha), p \uparrow \alpha\rangle$.

If $N$ belongs to $M$, then $N \subseteq M$, and since $\alpha \in[\bar{\beta}, \beta)$ this contradicts the fact that $\alpha \in N$. So $N$ must be outside $M$. It follows by properties of $s^{*}$ above that $N$ is either a node of $s$ or an intersection $N^{\prime} \cap W$, where $N^{\prime}$ is a small node in $s$ and $W \in \mathcal{T}$. If $N$ is a node of $s$, then $\langle s \cap H(\alpha), p \uparrow \alpha\rangle$ forces $p(\alpha)$ to be a master condition for $N$, because $\langle s, p\rangle$ is a condition in $\mathbb{A}_{\beta}$. The same is true in the case when $N$ has the form $N^{\prime} \cap W$ for $N^{\prime} \in s$ and $W \in \mathcal{T}$, using Remark 6.4. This completes the proof of the limit case of the lemma.

Suppose next that $\beta$ is a successor point of $Z$. Let $\alpha$ be the predecessor of $\beta$ in $Z$. By elementarity of $M^{*}, \alpha \in M^{*}$. For expository simplicity, fix $G$ which is generic for $\mathbb{A}_{\alpha}$ over $V$, with $\langle s, p \uparrow \alpha\rangle \in G$. By induction $\langle s, p \uparrow \alpha\rangle$ is a master condition for $M^{*}$ in $\mathbb{A}_{\alpha}$, so $M^{*}[G] \prec H\left(\theta^{*}\right)[G]$ and $M^{*}[G] \cap V=M^{*}$.

Suppose that $H(\alpha)$ is a node in $s$. (We will handle the case when $H(\alpha) \notin s$ later.) By Lemma 6.7, $G \cap H(\alpha)$ is generic for $\mathbb{A} \cap H(\alpha)$ over $V$.

If it is not forced in $\mathbb{A} \cap H(\alpha)$ that $F(\alpha)$ is a proper poset, then $\mathbb{A}_{\beta}$ is equal to $\mathbb{A}_{\alpha}$ and the lemma at $\beta$ follows immediately by induction. Suppose then that $F(\alpha)$ is forced to be a proper poset, and let $\mathbb{Q}=F(\alpha)[G \cap H(\alpha)]$. $\mathbb{Q}$ belongs to $M^{*}[G \cap H(\alpha)] \subseteq M^{*}[G]$.

Let $W$ be the first transitive node of $s$ above $H(\alpha)$ if there is one, and $H(\theta)$ otherwise. Let $N_{0} \in N_{1} \in \cdots \in N_{l-1}$ list the small nodes of $s$ between $H(\alpha)$ and $W$, in increasing order. Let $k<l$ be such that $N_{k}=M \cap W$. Note that the nodes in $\left\{N_{0}, \ldots, N_{l-1}\right\}$ that belong to $\operatorname{res}_{M}(s)$ are exactly the nodes $N_{0}, \ldots, N_{k-1}$.

Fix $D \in M^{*}$ which is dense in $\mathbb{A}_{\beta}$. Let $E$ be the set of $u \in \mathbb{Q}$ so that one of the following two conditions holds.

(i) No extension of $u$ is a master condition for the models $N_{i}[G \cap H(\alpha)]$ for all $i<k$.

(ii) There exists $\langle t, q\rangle \in D$ with $\langle t \cap H(\alpha), q \uparrow \alpha\rangle \in G, t \leq \operatorname{res}_{M}(s)$, and $q(\alpha)[G \cap H(\alpha)]=u$.

In the case of condition (ii), by Definition 6.1 and the fact that $t \leq \operatorname{res}_{M}(s), u$ is a master condition for $N_{i}[G \cap H(\alpha)]$ for all $i<k$.

All the parameters in the definition of $E$ belong to $M^{*}[G \cap H(\alpha)]$, and therefore by elementarity so does $E$. The density of $D$ in $\mathbb{A}_{\beta}$ implies that $E$ is dense in $\mathbb{Q}$. To see this, let $\bar{u}=\dot{\bar{u}}[G \cap H(\alpha)]$ be any condition in $\mathbb{Q}$, and suppose for contradiction that it has no extension in $E$. Let $\langle a, h\rangle \in G \cap H(\alpha)$ force this. Extending $\bar{u}$ using failure of (i) we may assume that it is a master condition for $N_{i}[G \cap H(\alpha)]$ for all $i<k$. We may assume that $\langle a, h\rangle$ forces this by extending $\langle a, h\rangle$ if needed, and with further extension we may assume also that $\operatorname{res}_{M}(s) \cap H(\alpha) \subseteq a$, since $\left\langle\operatorname{res}_{M}(s), \varnothing\right\rangle \in G$. Let $a^{*}=a \cup \operatorname{res}_{M}(s)$ (which below $W$ is exactly $\left.a \cup\left\{H(\alpha), N_{0}, \ldots, N_{k-1}\right\}\right)$. Let $h^{*}=h \cup\{\alpha \mapsto \dot{\bar{u}}\}$. Then $\left\langle a^{*}, h^{*}\right\rangle$ is 
a condition in $\mathbb{A}_{\beta}$, and any $\langle t, q\rangle \in D$ which extends it provides a contradiction to the fact that $\dot{\bar{u}}$ is forced to have no extensions in $E$.

We proceed by using the density of $E$, and the properness of $\mathbb{Q}$, to prove condition (1) of the lemma.

If $\alpha \in \operatorname{dom}(p)$, then by Definition 6.1, $p(\alpha)[G \cap H(\alpha)]$ is a master condition for $M^{*}[G \cap H(\alpha)]$ in $\mathbb{Q}$. It follows using the density of $E$ that there is $u \in E \cap M^{*}[G \cap H(\alpha)]$, and $u^{*}$ which extends both $p(\alpha)[G \cap H(\alpha)]$ and $u$. Since $u^{*}$ extends $p(\alpha)[G \cap H(\alpha)]$, it is a master condition for $N_{i}[G \cap H(\alpha)]$ for all $i<l$, and equivalently for all small nodes $N$ of $s$ with $\alpha \in N$.

If $\alpha \notin \operatorname{dom}(p)$, then $u$ and $u^{*}$ with the same properties can be obtained as follows. First let $v \in \mathbb{Q}$ be a master condition for $N_{i}[G \cap H(\alpha)]$ for all $i<l$. This is possible using Claim 6.8. Then obtain $u$ and $u^{*}$ as in the previous paragraph, starting from $v$ instead of $p(\alpha)[G \cap H(\alpha)]$.

Since $u^{*} \leq u$ is a master condition for each $N_{i}[G \cap H(\alpha)]$, the membership of $u$ in $E$ must hold through condition (ii) in the definition of $E$. Let $\langle t, q\rangle$ witness the condition. Using the elementarity of $M^{*}[G \cap H(\alpha)]$, pick $\langle t, q\rangle$ in this model. Since $M^{*}[G \cap H(\alpha)] \cap V=M^{*},\langle t, q\rangle$ belongs to $M^{*}$.

Then $\langle t, q\rangle$ is a condition in $\mathbb{A}_{\beta}$, and in particular $q(\alpha)$ is forced by $\langle t \cap H(\alpha)$, $q \nmid \alpha\rangle$ to be a master condition for all small nodes $N \in t$ with $\alpha \in N$. Since $q(\alpha)[G \cap H(\alpha)]=u$ and $\langle t \cap H(\alpha), q \uparrow \alpha\rangle \in G, u$ is a master condition for these nodes.

By Corollary 2.31, $t$ and $s$ are directly compatible. Let $r$ witness this. By the same corollary, $\operatorname{res}_{M}(r)=t$, and hence the small nodes of $r$ inside $M$ are nodes of $t$. Again by the corollary, the small nodes of $r$ outside $M$ are either nodes of $s$ or intersections of small nodes of $s$ with transitive nodes of $t$. Since $u$ is a master condition for small $N \in t$ with $\alpha \in N$, and $u^{*} \leq u$ is a master condition for small $N \in s$ with $\alpha \in N$, it follows using Remark 6.4 that $u^{*}$ is a master condition for all $N \in r$ with $\alpha \in N$.

Let $\dot{u}$ and $\dot{u}^{*}$ name $u$ and $u^{*}$, respectively. Let $\langle a, h\rangle \in G \cap H(\alpha)$ be strong enough to force all the properties of $u, u^{*}$, and $\langle t, q\rangle$ proved above. Extending $\langle a, h\rangle$ we may assume it is stronger than both $\langle s \cap H(\alpha), p \nmid \alpha\rangle$ and $\langle t \cap H(\alpha), q \uparrow \alpha\rangle$.

By Claim 2.33, $r \cap H(\alpha)=\operatorname{res}_{H(\alpha)}(r)$ is the closure of $\operatorname{res}_{H(\alpha)}(s) \cup \operatorname{res}_{H(\alpha)}(t)=$ $(s \cup t) \cap H(\alpha)$ under intersections. Since $\langle a, h\rangle$ extends both $\langle s \cap H(\alpha), p \nmid \alpha\rangle$ and $\langle t \cap H(\alpha), q \uparrow \alpha\rangle$, and since $a$ is closed under intersections, $r \cap H(\alpha) \subseteq a$. By Corollary 2.31, $a$ and $r$ are then directly compatible, and this is witnessed by $a \cup r$.

Let $s^{*}=a \cup r$. Let $p^{*}=h \cup\left\{\alpha \mapsto \dot{u}^{*}\right\}$. It is easy now to check that $\left\langle s^{*}, p^{*}\right\rangle$ is a condition in $\mathbb{A}_{\beta}$ and extends both $\langle s, p\rangle$ and $\langle t, q\rangle$. This, together with properties of $r$ and $a$ proved above, completes the proof of condition (1) in the case when $\beta$ is a successor, $\alpha$ is the predecessor of $\beta$ in $Z$, and $H(\alpha) \in s$.

Suppose finally that $\beta$ is a successor point of $Z, \alpha$ is the predecessor of $\beta$ in $Z$, and $H(\alpha) \notin s$. By Claim 5.7 and Remark 5.8, there is an extension $s^{\prime}$ of $s$ with $H(\alpha) \in s^{\prime}$, so that the only added nodes, meaning nodes in $s^{\prime}-s$, are transitive nodes and intersections of small nodes of $s$ with transitive nodes. Then using Remark 6.4, $\left\langle s^{\prime}, p\right\rangle$ is a condition. By the arguments above the lemma holds for $\left\langle s^{\prime}, p\right\rangle$, and this implies that it holds for $\langle s, p\rangle$.

Corollary 6.13 Forcing with $\mathbb{A}$ preserves $\omega_{1}$ and $\theta$ as cardinals. All cardinals between $\omega_{1}$ and $\theta$ are collapsed to $\omega_{1}$. 
Proof Preservation of $\theta$ is immediate from the strong properness given by Lemma 6.7 and stationarity of $\mathcal{T}$. Preservation of $\omega_{1}$ is immediate from the properness given by Lemma 6.12 for $\mathbb{A}=\mathbb{A}_{\theta}$, Corollary 6.11 , and stationarity of $\delta$. The arguments in Section 5.1 applied to the sequence poset component of $\mathbb{A}$ show that all cardinals between $\omega_{1}$ and $\theta$ are collapsed to $\omega_{1}$.

Lemma 6.14 The extension of $V$ by $\mathbb{A}$ satisfies the proper forcing axiom.

Proof This follows from the inclusion of a Laver iteration of proper posets in $\mathbb{A}$. The argument is standard but uses the strong properness given by Lemma 6.7 rather than the automatic strong properness that holds for iterations. We give a brief sketch.

Suppose that the lemma fails, and let $\langle a, h\rangle \in \mathbb{A}$ force that $\dot{\mathbb{Q}}$ and $\dot{D}_{\xi}, \xi<\omega_{1}$, provide a counterexample. Pick $\dot{\mathbb{Q}}$ so that it is outright forced to be proper. Let $\gamma$ be large enough that $\dot{\mathbb{Q}}$ and $\dot{D}_{\xi}$ belong to $H(\gamma)$. Since $F$ is a Laver function and $\theta$ is supercompact, there are $\bar{\theta}<\theta, \bar{\gamma}<\theta, \dot{\mathbb{P}} \in H(\bar{\gamma})$, and $\dot{E}_{\xi} \in H(\bar{\gamma})$ for $\xi<\omega_{1}$, so that

(*) $\left(H(\bar{\gamma}) ; F \uparrow \bar{\theta}, \dot{\mathbb{P}}, \dot{E}_{\xi}\right)$ embeds elementarily into $\left(H(\gamma) ; F, \dot{\mathbb{Q}}, \dot{D}_{\xi}\right)$ via an embedding, $\pi$, say, with critical point $\bar{\theta}$, and $F(\bar{\theta})=\dot{\mathbb{P}}$.

(To see that a structure and embedding witnessing $(*)$ exist note that by standard use of the Laver function there is $\sigma: V \rightarrow M$ with $\sigma(F)(\theta)=\dot{\mathbb{Q}}$ and $\sigma \uparrow H(\gamma) \in M$. Then in $M$ there exist a structure, namely, $\left(H(\gamma) ; F, \dot{\mathbb{Q}}, \dot{D}_{\xi}\right)$, and an embedding, namely, $\sigma \uparrow H(\gamma)$, which satisfy $(*)$ relative to $\sigma\left(H(\gamma) ; F, \dot{\mathbb{Q}}, \dot{D}_{\xi}\right)$. Pulling back using the elementarity of $\sigma$ gives the required structure and embedding in $V$.)

$\bar{\theta}$ may be picked large enough that $\langle a, h\rangle \in \mathbb{A} \cap H(\bar{\theta})$. Then by Lemma 6.7, $\langle a \cup\{H(\bar{\theta})\}, h\rangle$ is a condition in $\mathbb{A}$, and a strong master condition for $H(\bar{\theta})$.

Let $G$ be generic for $\mathbb{A}$ with $\langle a \cup\{H(\bar{\theta})\}, h\rangle \in G$. By strong properness, $G \cap H(\bar{\theta})$ is generic for $\mathbb{A} \cap H(\bar{\theta})$ over $V$, and $\pi$ extends trivially to an embedding of $H(\bar{\gamma})[G \cap H(\bar{\theta})]$ into $H(\gamma)[G]$.

It is easy to check, from the definition of $\mathbb{A}$, genericity of $G$, and the fact that $F(\bar{\theta})=\dot{\mathbb{P}}$, that $\{p(\bar{\theta})[G \cap H(\bar{\theta})] \mid\langle s, p\rangle \in G$ and $\bar{\theta} \in \operatorname{dom}(p)\}$ is generic for $\dot{\mathbb{P}}[G \cap H(\bar{\theta})]$ over $V[G \cap H(\bar{\theta})]$. The image of this set under the extended embedding $\pi$ is a filter on $\mathbb{Q}=\dot{\mathbb{Q}}[G]$ that meets each of the sets $D_{\xi}=\dot{D}_{\xi}[G]$.

\section{References}

[1] Caicedo, A. E., and B. Veličković. "The bounded proper forcing axiom and well orderings of the reals," Mathematics Research Letters, vol. 13 (2006), pp. 393-408. MR 2231126. DOI 10.4310/MRL.2006.v13.n3.a5. 289

[2] Friedman, S.-D., "Forcing with finite conditions," pp. 285-95 in Set Theory, Trends in Mathematics, Birkhäuser, Basel, 2006. MR 2267153. DOI 10.1007/3-7643-7692-9_10. 265, 283, 285, 286, 289

[3] Friedman, S.-D., "BPFA and inner models," Annals of the Japan Association for Philosophy of Science, vol. 19 (2011), pp. 29-36. MR 2857735. 266, 278, 280, 283, 289, 290

[4] Friedman, S.-D., and J. Krueger. "Thin stationary sets and disjoint club sequences," Transactions of the American Mathematical Society, vol. 359 (2007), pp. 2407-20. MR 2276627. DOI 10.1090/S0002-9947-06-04163-8. 286

[5] Jech, T., Set Theory, 3rd millennium edition, revised and expanded, Springer Monographs in Mathematics, Springer, Berlin, 2003. MR 1940513. 284 
[6] Mitchell, W. J., "Adding closed unbounded subsets of $\omega_{2}$ with finite forcing," Notre Dame Journal of Formal Logic, vol. 46 (2005), pp. 357-71. MR 2162106. DOI 10.1305/ndjfl/1125409334. 265, 266, 278, 283, 285, 286

[7] Mitchell, W. J., " $I\left[\omega_{2}\right]$ can be the nonstationary ideal on $\operatorname{Cof}\left(\omega_{1}\right)$," Transactions of the American Mathematical Society, vol. 361 (2009), pp. 561-601. MR 2452816. DOI 10.1090/S0002-9947-08-04664-3. 267

[8] Neeman, I., "Aronszajn trees and failure of the singular cardinal hypothesis," Journal of Mathematical Logic, vol. 9 (2009), 139-57, 2009. MR 2665784. DOI 10.1142/S021906130900080X. 288

[9] Neeman, I., "Forcing with side conditions," conference lecture at Oberwolfach, Germany, 2011, http: / /www. math.ucla.edu/ ineeman/fwsc.pdf/. 266

[10] Sinapova, D., "The tree property and the failure of the singular cardinal hypothesis at $\aleph_{\omega^{2}}, "$ Journal of Symbolic Logic, vol. 77 (2012), pp. 934-46. MR 2987144. DOI 10.2178/jsl/1344862168. 288

[11] Todorčević, S., Partition Problems in Topology, vol. 84 of Contemporary Mathematics, American Mathematical Society, Providence, 1989. MR 0980949. DOI 10.1090/conm/084. 265

[12] Veličković, B., "Forcing axioms and stationary sets," Advances in Mathematics, vol. 94 (1992), pp. 256-84. MR 1174395. DOI 10.1016/0001-8708(92)90038-M. 289

[13] Veličković, B., and G. Venturi, "Proper forcing remastered," pp. 331-362 in Appalachian Set Theory, 2006-2012, vol. 406 of London Mathematical Society Lecture Note Series, Cambridge University Press, Cambridge, 2012. 266

\section{Acknowledgment}

This material is based upon work supported by the National Science Foundation under grants DMS-0556223 and DMS-1101204.

Department of Mathematics

University of California, Los Angeles

Los Angeles, California 90095-1555

USA

ineeman@math.ucla.edu 